\title{
Foundations of Advanced Magnetic Resonance Imaging
}

\author{
Roland Bammer,* Stefan Skare, ${ }^{*}$ Rexford Newbould, ${ }^{*}$ Chunlei Liu, ${ }^{*}$ Vincent Thijs, ${ }^{\dagger}$ \\ Stefan Ropele, ${ }^{\ddagger}$ David B. Clayton, ${ }^{*}$ Gunnar Krueger,${ }^{\S}$ Michael E. Moseley, $*$ \\ and Gary H. Glover* \\ *Lucas MRS/I Center, Department of Radiology, Stanford University, California; ${ }^{\dagger}$ Department of Neurology, UZ Gasthuisberg, \\ B-3000 Leuven, Belgium; ${ }^{\ddagger}$ MR Institute, Medical University Graz, 8036 Graz, Austria; and ${ }^{\S}$ Siemens Medical Solutions, \\ Department of MREA, 91052 Erlangen, Germany
}

\begin{abstract}
Summary: During the past decade, major breakthroughs in magnetic resonance imaging (MRI) quality were made by means of quantum leaps in scanner hardware and pulse sequences. Some advanced MRI techniques have truly revolutionized the detection of disease states and MRI can nowwithin a few minutes-acquire important quantitative information noninvasively from an individual in any plane or
\end{abstract}

volume at comparatively high resolution. This article provides an overview of the most common advanced MRI methods including diffusion MRI, perfusion MRI, functional MRI, and the strengths and weaknesses of MRI at high magnetic field strengths. Key Words: Diffusion, perfusion, spin labeling, diffusion tensor, functional MRI, tractography.

\section{INTRODUCTION}

Magnetic resonance imaging (MRI) has been established for over a decade as a superior research and clinical modality for anatomical imaging. Noteworthy for exceptionally good submillimeter spatial and subsecond temporal resolution, MRI is now demonstrating the potential of tracing the links between tissue function, metabolism, blood flow, and hemodynamics in both normal and disease states. Likewise, MRI can-within minutes-noninvasively acquire functional images in any plane or volume at comparatively high resolution. Functional MRI (fMRI) can image the hemodynamic and metabolic changes that are associated with human brain functions, such as vision, motor skills, language, memory, and mental processes. These techniques have also revolutionized detection of a wide variety of disease states, such as stroke, multiple sclerosis, and tumors.

Much of the recent progress in MRI resulted because of high-speed imaging capabilities. This typically means that enhanced gradient strengths are used to produce images in one or a few shots or echo-trains. Echo-planar

Address correspondence and reprint requests to Roland Bammer, Ph.D., Lucas MRS/I Center, Department of Radiology, Stanford University, 1201 Welch Road, Stanford, CA 94305-5488. E-mail: rbammer@stanford.edu. and spiral imaging are two variations of such high-speed gradient-echo MRI techniques, whereas spin-echo variations of high-speed imaging fall into the class of fast spin-echo MRI. High-speed MRI suggests that images are acquired in several seconds or fewer and provide the means of producing motion-free images or a series of rapidly acquired images.

In this review article, we will review the foundations of these advanced MRI techniques and will attempt to address their potential strengths and weaknesses. Furthermore, we will provide an overview of the potential applications of advanced MRI in neurologic diagnosis and therapeutic monitoring.

\section{DIFFUSION MRI}

Diffusion sets the basis for one of the most robust contrast mechanisms in MRI. ${ }^{1}$ Diffusion-weighted MRI (DWI) and more advanced methods, such as diffusion tensor imaging (DTI), ${ }^{2,3}$ have advanced from an experimental tool to a frequently used method for clinical evaluation and for a better understanding of the pathophysiology of certain diseases. Specifically, DWI now belongs to the standard arsenal of MRI pulses sequences, and the impact it has made for the early diagnosis of acute stroke ${ }^{4,5}$ seems to be unmatched by other MRI 


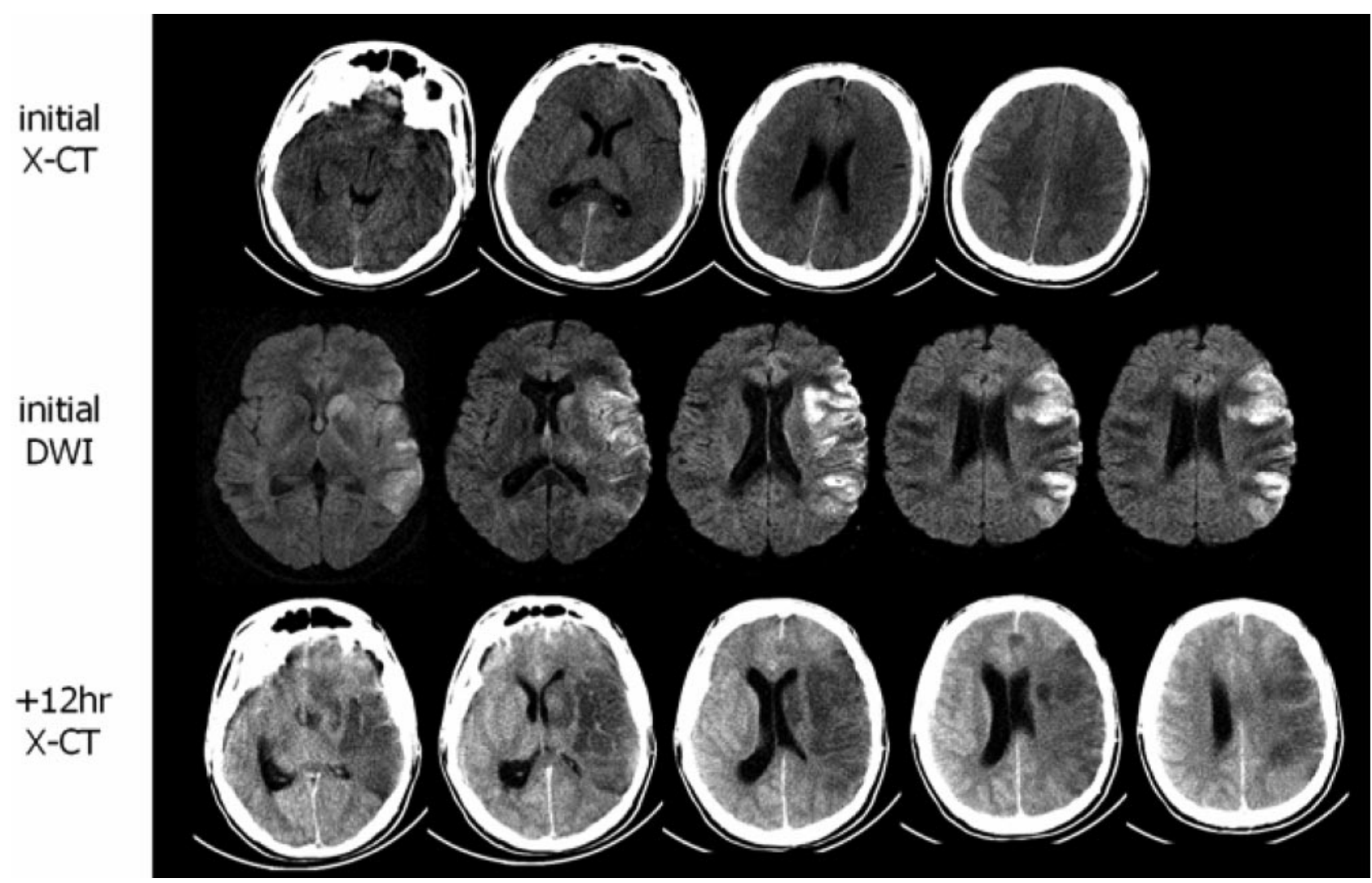

FIG. 1. Acute ischemic stroke of an 18-year-old man. Top row: Approximately $1.5-2 \mathrm{~h}$ after onset of clinical symptoms, conventional CT shows no clear signs of infarction. Middle row: Series of diffusion-weighted images obtained immediately after CT allows exact delineation of injured tissue. Bottom row: Follow-up CT clearly confirms findings on DWI examination. DWI was performed with a navigated diffusion-weighted interleaved EPI sequence.

methods. The possibility of diagnosing stroke within the window of opportunity to treat patients while brain tissue is still salvageable and to triage patients with different etiology of a stroke is of great importance for treatment decisions in critically ill patients (FIG. 1). ${ }^{6}$

Although diffusion tensor imaging has not yet been able to match the impact of DWI in stroke, it has proven to be a sensitive tool for detecting subtle abnormalities in the white matter of patients with diseases like multiple sclerosis $^{7,8}$ or ALS. ${ }^{9}$ Despite DTI's sensitivity to white matter abnormalities shown in group comparisons, further work in this area is warranted to demonstrate its value for diagnosing individual patients.

The anisotropic nature of water diffusion in white matter has led yet to another exciting modality that allows investigators to follow different fiber systems in white matter. ${ }^{10}$ Despite its appearance on conventional T1-weighted series, white matter is not a compact mass of myelinated axons supported by glia cells and blood vessels. Rather, it consists of highly organized and numerous fiber systems, each of which interconnects with different areas at the cortex, deep gray matter structures, and the periphery. Fiber tracking, based on orientational information obtained from DTI or more sophisticated methods, promises to elucidate, at least at the gross mor- phological level, functional and anatomical connectivity in the brain.

\section{Basic concept of diffusion-weighted MRI}

MRI allows one to measure the self-diffusion of water- that is, the Brownian or random motion of the water molecules driven by their internal thermal energy. ${ }^{1}$ By means of DWI, it is possible to take snapshots of the water motion on a time scale of a few tens of milliseconds. Within pure water or CSF, diffusion is usually unrestricted and the measured diffusion constant is independent of the direction along which the apparent diffusion coefficient (ADC) was measured. The diffusion coefficient that is obtained from a tissue voxel via MRI reflects the contribution of different compartments. If diffusion is isotropic, there is no preferred direction of water motion for these tissues. However, for white matter, consisting of dense fiber bundles, water moves more easily parallel to the fibers than across them. Depending on the fiber- packing density the displacement front of the water protons will be more or less cigar shaped (i.e., a stretched ellipsoid) in contrast to a spherical shape for isotropic diffusion. Here, the orientation of its long axis will be dependent on the local fiber orientation (FIG. 2). The diffusion ellipsoid [i.e., a three-dimensional (3-D) 


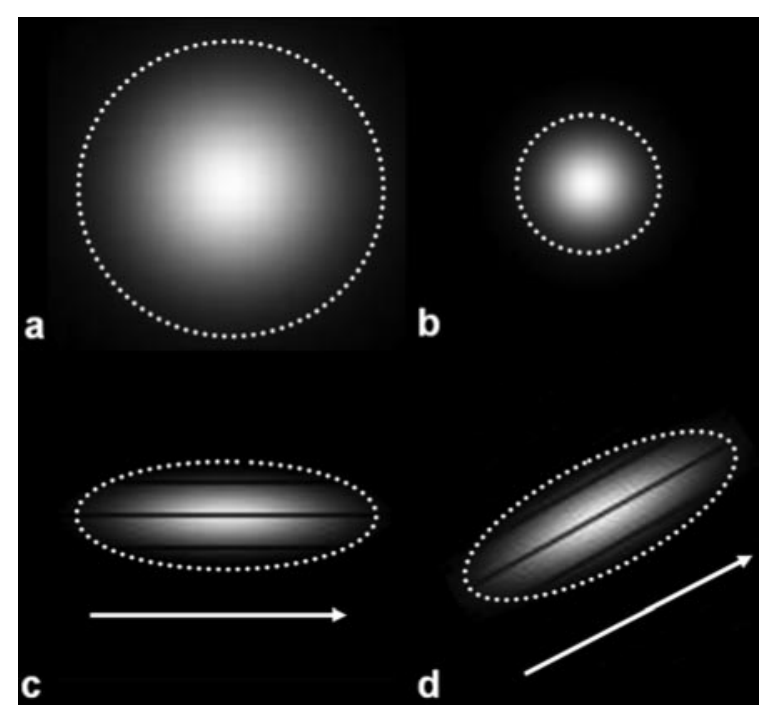

FIG. 2. Visualization of the proton displacement front due to diffusion. a: Isotropic diffusion occurs if diffusion is equal along all directions. b: For the same diffusion observation time interval, the proton displacement front (dotted line) is smaller in the presence of reduced diffusion. With anisotropic diffusion (c and d), diffusion differs along different directions.

ellipse] is parameterized with six elements. ${ }^{1}$ The first three parameters are the radii along the principal axes of the ellipsoid. If they are all equal, this would represent a sphere and, hence, isotropic diffusion. The other three parameters are the rotation angles of the ellipsoid defining its orientation in space, relative to the laboratory frame of reference.

\section{DIFFUSION TENSOR IMAGING}

Diffusion tensor imaging (DTI) is based on measurements of water diffusion along many different directions. These measurements allow one to calculate the diffusion tensor and, hence, the diffusion ellipsoids. Here, the diffusion tensor (D) is expressed by a diagonal symmetric 3 $\times 3$ matrix consisting of six unique elements. The tensor is the mathematical representation of the diffusion ellipsoid-one for each voxel. DWI scans with encoding along at least six directions are required to determine the six unknown parameters of the ellipsoid. ${ }^{2,3}$ However, the diffusion is often measured along many directions; a few tens of directions will already improve the image quality of the tensor estimations and will avoid orientational bias. Measuring diffusion along a certain direction is done by combining the $\mathrm{x}, \mathrm{y}$, and $\mathrm{z}$ magnetic field gradients that will lead to a net field gradient along the direction vector defined by $\left(G_{x}, G_{y} G_{z}\right)^{T}$. In the simplest form of DWI, these encoding gradients are added to a spin echo sequence and will lead to an observable signal attenuation that is related to the diffusivity of the protons (FIG. 3). The gradients have no effect on static protons. However, protons moving along the diffusion encoding
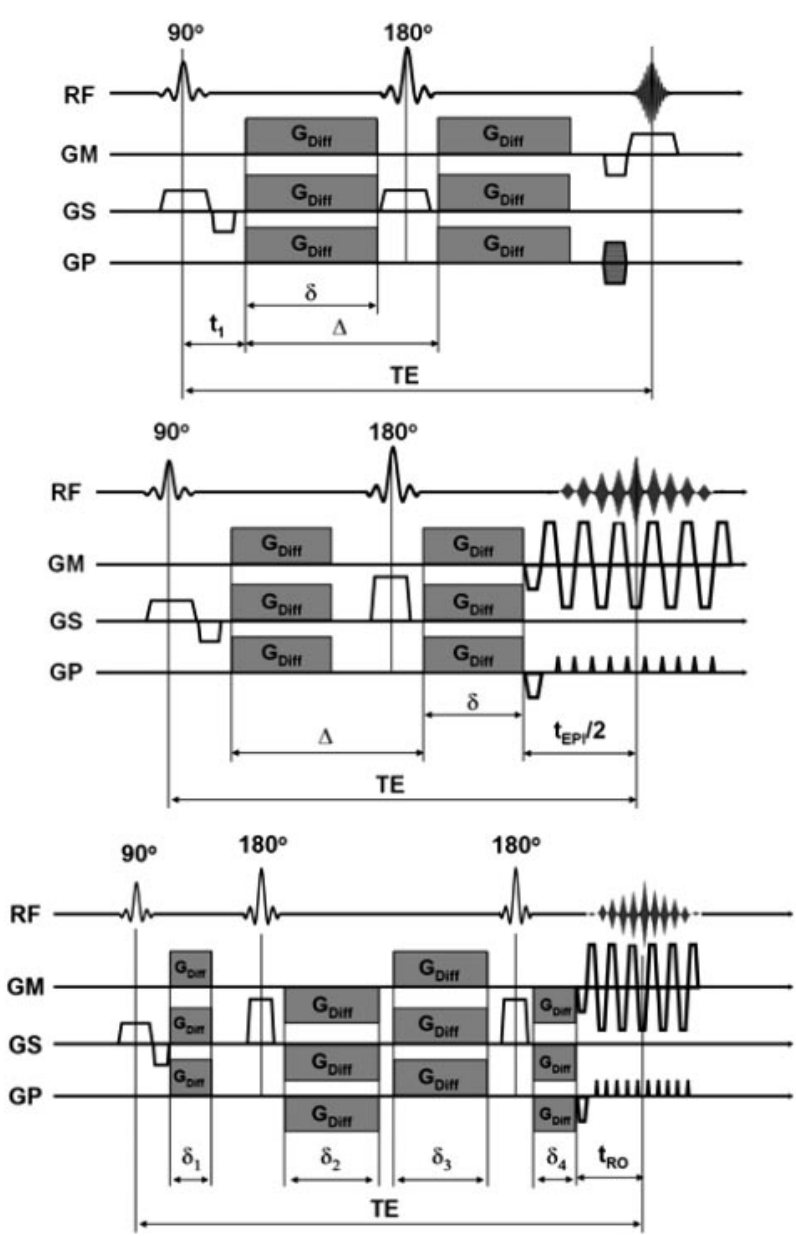

FIG. 3. a: Diffusion-weighted spin echo sequence: diffusionweighting gradients are applied during each TE/2 period. The diffusion-weighted echo is sampled at the time $t=T E$ when the spin echo is formed. The diffusion attenuation is only dependent on the parameters $G_{D i f f}, \Delta$, and $\delta$ but does not depend on $t_{1}$. $(\mathrm{GM}=$ readout direction, $\mathrm{GP}=$ phase-encode direction, and GS $=$ section select direction). b: Single-shot diffusion-weighted spin echo EPI pulse sequence. The entire $k$-space is filled with a single EPI-readout train. Because the EPI-train occupies much of the time available in the second TE/2 interval $\left(t_{E P} / 2\right)$, the maximum duration of the diffusion gradient lobes is mostly determined by the time TE/2- $t_{\mathrm{EPI}} / 2$. c: Twice-refocused dual spin echo diffusion-weighted pulse sequence. The RF pulses (excite and refocus), diffusion gradients $G$ of lengths $\delta_{1}, \delta_{2}, \delta_{3}$, and $\delta_{4}$, and an EPI readout are shown. Because the gradient pulses are shorter and have opposite polarity, the build-up of potent eddy currents is strongly reduced. Conversely, for the diffusing spins the bipolar gradient pair together with the refocusing RF pulse act as one long diffusion encoding gradient with efficient encoding power.

gradient direction will accrue a nonremovable phase during the diffusion observation interval. Due to the random motion pattern of each spin, this phase accrual will, on the macroscopic scale level, result in a net spin dephasing and loss of signal related to the extent of diffusivity within the voxel.

In Figure 4, three diffusion-weighted images are presented, with diffusion encoding gradients applied along the $x, y$, and $z$ direction. In observing white matter areas, 


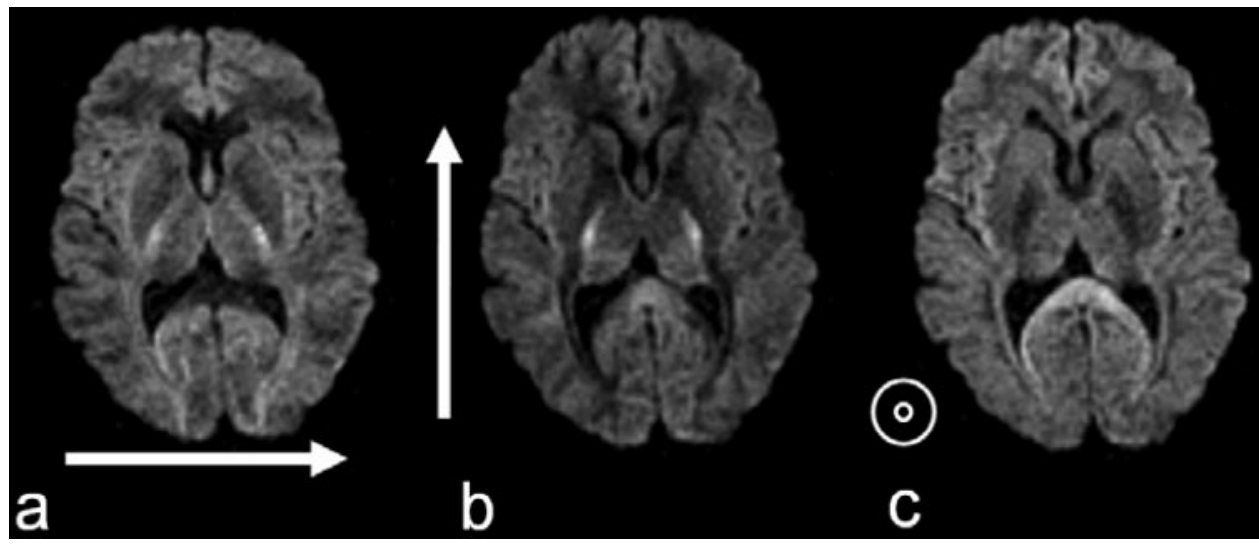

FIG. 4. Diffusion-weighted images with diffusion-encoding along the (a) left-right, (b) anterior-posterior, and (c) caudo-cranial direction. Notice that white matter fibers running perpendicular to the gradient direction appear hyperintense because of lower values of diffusivity, whereas fibers running parallel to the gradient direction demonstrate lower signal intensities.

such as the genu or splenium of the posterior corpus callosum, one can appreciate the signal difference caused by anisotropic diffusion. White matter structures appear hypointense relative to the surrounding tissue if the white matter tracts run parallel to the encoding gradient applied, whereas they appear hyperintense when fibers run perpendicular to the encoding gradient. In the first case, this is caused by a relatively high diffusion coefficient, whereas in the second case restricted or hindered diffusion occurs across multiple layers of oriented fibers.

From the six or more DW images and the diffusion gradient directions being used, the elements of $\mathrm{D}$ can be calculated by simple matrix calculus. Unfortunately, neither the diffusion tensor element images nor some grid of ellipsoids is an adequate form that could be presented to the clinician for diagnosis because there is too much information to perceive at once. To interpret the tensor information, it is necessary to break down this information into single-valued measures that can be viewed as readable gray-scale maps. Fortunately, one can identify two relevant measures from the diffusion ellipsoid. The first is the mean diffusivity $(\langle D>)$, which is calculated by taking the average of the three radii of the ellipsoids. Mean diffusivity is measured in $\mathrm{mm}^{2} / \mathrm{s}$, as a consequence of the random motion with no net transport. The second measure, anisotropy, characterizes how much the diffusion ellipsoid deviates from a spherical shape. There are several measures that characterize this property, ${ }^{11}$ of which the relative anisotropy (RA) measure may be the most intuitive. RA is dimensionless as are all other measures of anisotropy. It is zero for isotropic diffusion (sphere shape) and approaches one for more pronounced cigar shapes.

To calculate these measures, the diffusion tensor D is first diagonalized, which is a standard mathematical operation on a matrix that essentially rotates the coordinate frame of reference independently (pixel-by-pixel) into the principal axes of the ellipsoid. By this, the three radii (or eigenvalues $\lambda_{1}, \lambda_{2}, \lambda_{3}$ ), as well as the orientation of the diffusion ellipsoid, are explicitly obtained. The mean diffusivity is then calculated by

$$
\langle\mathrm{D}\rangle=\left(\lambda_{1}+\lambda_{2}+\lambda_{3}\right) / 3
$$

and the RA as

$$
\mathrm{RA}=\frac{\sigma\left(\lambda_{1}, \lambda_{2}, \lambda_{3}\right)}{\langle\mathrm{D}\rangle}
$$

The RA is the standard deviation of the radii divided by their mean. This processing queue is illustrated in Figure 5. Notice that the images on the diagonal are actually different before and after the diagonalization. The eigenvectors provide information about the direction of the maximum diffusion within a voxel upon which fiber tracking ${ }^{10}$ will be built.

In addition to the DWI spin echo sequence, both stimulated echo ${ }^{12}$ and steady-state free precession sequences can be used. ${ }^{13-15}$ It is beyond the scope of this manuscript to provide more details about these techniques; a more detailed description can be found elsewhere. ${ }^{16}$

\section{Motion problems in DWI}

Current methods of diffusion preparation impart a spatially varying set of phases to tissue by means of diffusion-encoding gradients and then attempt to remove these phases after an elapsed period of time. Motion in the tissue sample, including bulk motion, during this time, often just a few tens of milliseconds, will leave localized phase on the object being imaged. Even in cooperative patients, peristalsis, respiratory motion, and pulsatile flow will confound diffusion imaging. ${ }^{17,18}$ Here, one must distinguish between coherent motion, which leads to a constant phase accrual for all spins, and incoherent motion (diffusion, microperfusion, turbulent flow), which causes spins with different magnitude and direction of motion (flow) to accrue different phase 


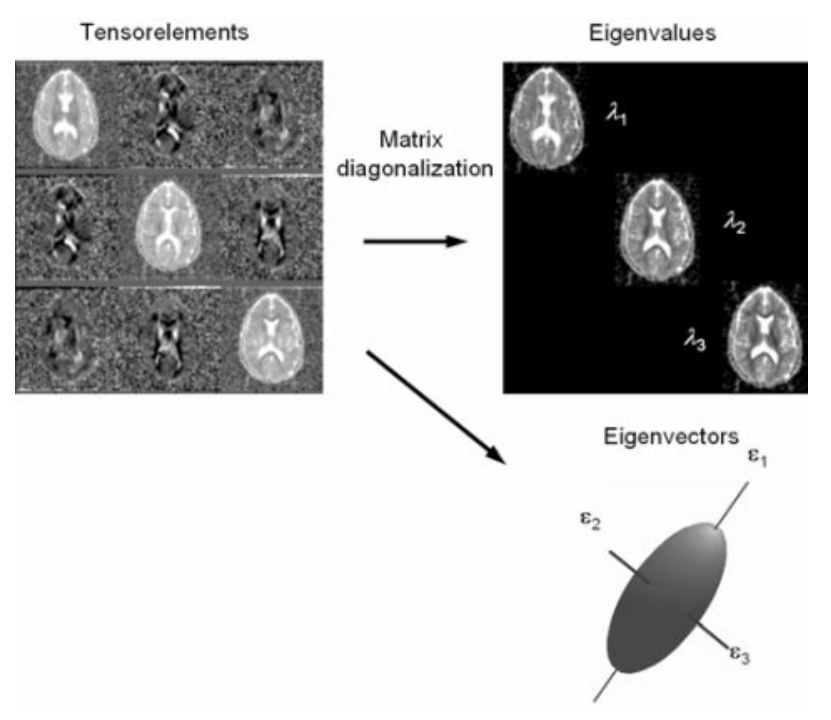

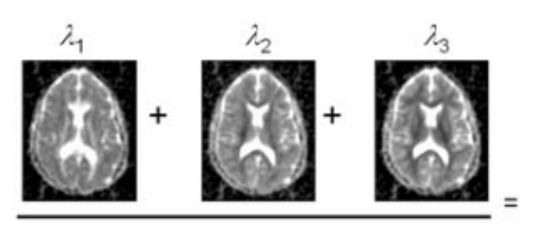

3

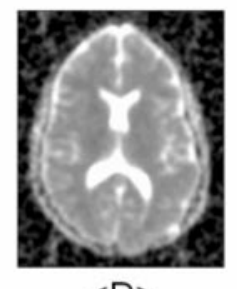

$<\mathrm{D}>$
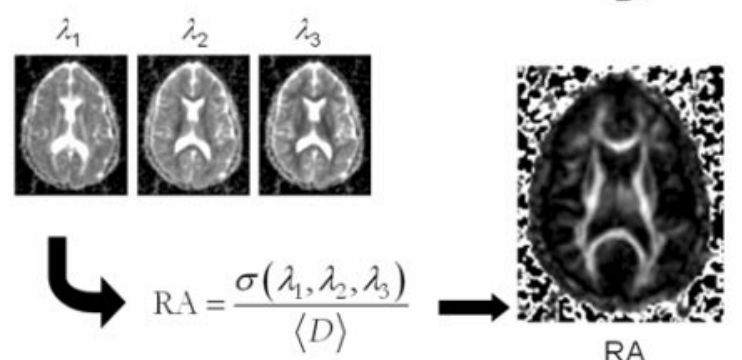

RA

FIG. 5. Processing of diffusion tensor information. a: From at least six DWls with diffusion-encoding along noncollinear direction plus one reference image, one can compute the six independent elements of the diffusion tensor (left). Notice that $D_{i j}=$ $D_{j i}$. By means of matrix diagonalization the eigenvalues (top right) and eigenvectors (top bottom) of the tensor can be computed. b: From the eigenvalues scalar, rotational invariant measures, i.e., trace of the diffusion tensor $\angle D>$ (top) and relative anisotropy (RA, bottom) can be computed.

terms. Because DWI is sensitive to motion on the order of tens of micrometers, even locations not commonly noted for motion, such as the brain, move orders of magnitude more than the diffusive motion under investigation, and such motions can corrupt image quality. This effect can mimic increased diffusivity and yield diffusion coefficients that are higher than the true underlying values. In other words, the root-mean-square displacement of diffusing spins usually covers an area of approximately $1 \mu \mathrm{m}^{2} / \mathrm{s}$; any type of macroscopic motion may therefore severely disturb measurements that are sensitive to microscopic movement. To address this issue, DWI is often acquired with cardiac triggering, i.e., synchronized with the cardiac cycle so that imaging during systole can be avoided.

In addition to erroneous diffusion measurements, bulk motion during the presence of strong diffusion-encoding gradients can also cause artifacts. In MRI, $k$-space data are normally acquired line by line or by a subset of lines. Several acquisitions (multishot) are therefore required to form a fully encoded image. This poses two problems for DTI: firstly, to compute diffusion tensor information, one must acquire at least six DWIs plus one $\left(\mathrm{T}_{2}\right.$-weighted $)$ reference image, which can be time consuming; secondly, and more importantly, bulk motion during activated diffusion encoding gradients leads to random phase accruals that can differ from shot to shot and compete with the phase information imprinted on the spins by the regular phase encoding. ${ }^{19,20}$ This additional perturbation will alter the true location and phase of the data in $k$-space and usually leads to significant ghosting (FIG. 6a). In this context, rigid body motion, comprised translation and/or rotation of the sample, cause detectable and potentially correctable phase errors. Rigid body translations during the application of the diffusion gradients introduce an additional constant phase offset to the data. Translations between readouts in a multishot acquisition will add a linear phase ramp across the line of data, whereas rotation shifts the $k$-space data in at least two directions relative to the sampling locations (FIG. 6, b-e). These phase errors can be detected or corrected by a number of means, including collecting additional scan data (known as navigator echoes) at the expense of scanning time. It should be noted that nonrigid body motions, such as plastic deformation of tissue, incur quite complex changes in the detected signal and are, in general, not handled.

\section{Navigator correction}

Multishot sequences have been commonly used to improve the resolution of DWI, but they generally suffer from shot-to-shot phase variations caused by motion. Whereas these phase terms yield to no consequences for single-shot echo planar imaging (EPI), this is a more severe problem in an interleaved acquisition. To capitalize on the better spatial resolution of multishot scans, motion-induced phase errors must be removed from each separate acquisition. ${ }^{20-24}$ Two-dimensional phase correction is currently the most commonly used method; it can be achieved by either acquiring extra data around the center of the $k$-space before image acquisition or by applying specially designed readout trajectories that fully sample the center of the $k$-space at each acquisition. With the fully sampled navigator data, two techniques can then be applied to measure acquisition-to-acquisition motion: 

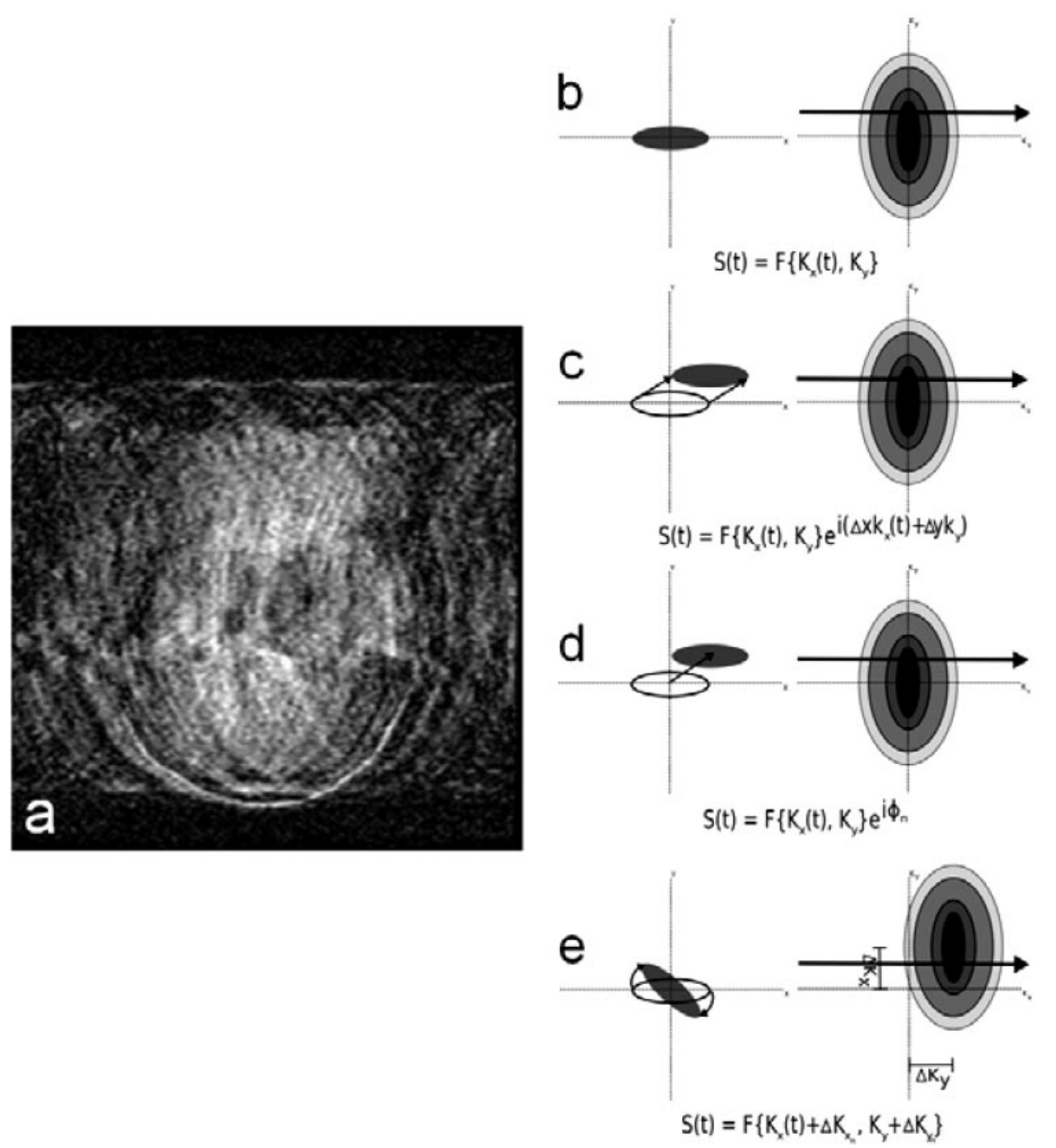

FIG. 6. a: Without any correction, motion in the presence of strong diffusion-weighting gradients results in severe image degradation. b-e (left column, image space; right column, $k$-space): A simplified representation of image space and $k$-space for a simple object. The readout is shown in a heavy black line. b: No rigid body motion is present; c: effect of translation between acquisitions; d: effect of translation during diffusion gradients; e: in-plane rotation during the application of the diffusion gradients.

one is to find the linear shift in $k$-space; the other is to find the phase variations in the image space. As described by both Anderson and Gore ${ }^{19}$ and Butts et al., ${ }^{20}$ motion-induced phase accrual causes the readout trajectory to deviate from the intended pathway and not start from the origin of $k$-space. The $k$-space approach estimates this $k$-space displacement from the navigator data and corrects for it before gridding reconstruction. ${ }^{25}$ The image-based technique estimates the phase error from the low-resolution image acquired by the navigator data and corrects for it in the image space for each acquisition. The purpose of both correction algorithms is to correct for the $k$-space displacement. As illustrated in Figure 7, the corrected $k$-space data show that after correction the peak of the signal is brought back to the origin of $k$-space.

The image-based phase correction also corrects for the nonlinear phase that exists in the low-resolution phase

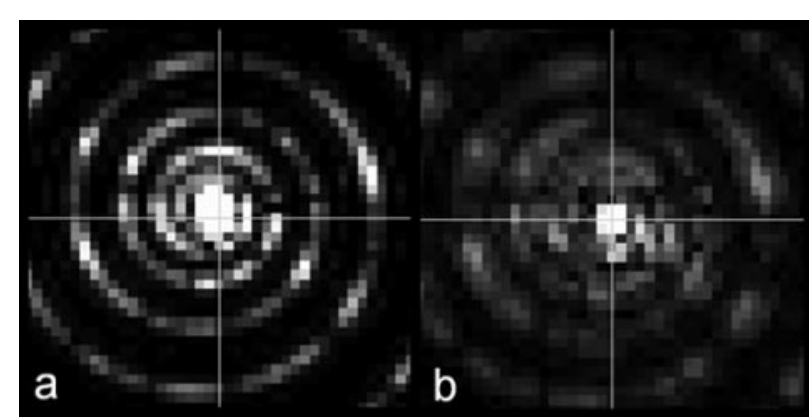

FIG. 7. Comparison of the $k$-space data before and after imagebased phase correction for one particular interleaf. Two white lines indicate the coordinate system. a: $k$-space data before correction. The data are displaced due to motion during the diffusion sensitizing gradients. b: $k$-space data after correction. The peak has been shifted back to the origin. Also notice that the intensity has been modified slightly because some nonlinear phase was corrected in the image space. 
a)

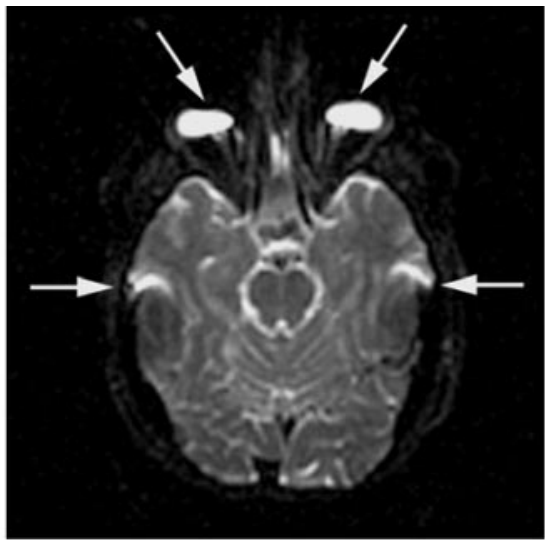

b)

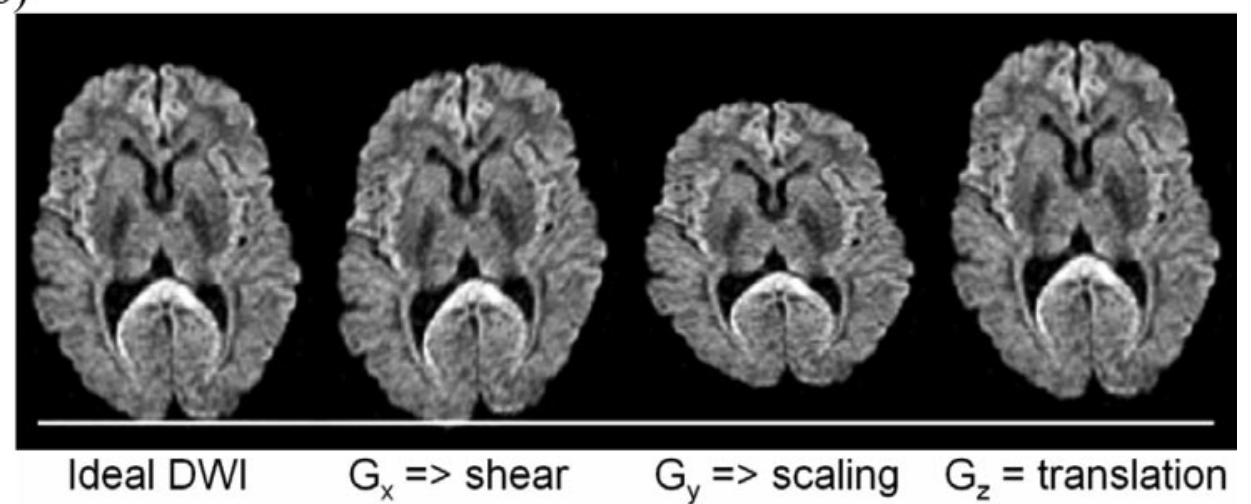

FIG. 8. a: EPI is generally prone to distortions from susceptibility differences (arrows). b: Because of the small bandwidth per pixel eddy current induced gradients can cause significant distortions along the phase encoding direction. Depending on the diffusion-encoding gradient orientation this can lead to either sheared, scaled, or translated images.

estimate. Alternatively, one can correct solely for the linear phase by extracting a linear phase term from the low-resolution image. However, Miller et al. ${ }^{26}$ showed that nonlinear phase correction can further restore signal loss and improve image quality because nonlinear phase in DWI can arise from nonrigid motion.

\section{Pulse sequences for DWI}

Diffusion-weighted single-shot EPI. Fast, single-shot methods such as EPI, ${ }^{27}$ combined with cardiac or respiratory gating, are less sensitive to motion and therefore minimize or even entirely avoid ghosting artifacts. Until recently, single-shot imaging techniques are almost exclusively used in the clinical routine. Using single-shot EPI, each image is acquired within the time frame of $0.1 \mathrm{~s}$. However, EPI suffers from its own problems: EPI is limited by lower resolutions $(\sim 128 \times 128)$, image blurring, localized signal loss, and image distortions. ${ }^{28}$ Distortions and signal loss occur predominantly at boundaries between tissue and air, due to the local change of magnetic field strength (FIG. 8). The degree of these distortions is dependent on both hardware and soft- ware. To minimize these artifacts, several acquisition methods ${ }^{29-31}$ and postprocessing methods have been proposed. $^{32-34}$

Yet another effect that impairs image quality in DWI with EPI is the distortion caused by eddy currents. Eddy currents are unwanted currents in conducting elements of the MRI system, usually in the Dewar of the magnet and are caused by the switching of the usually strong, lengthy diffusion-encoding gradients. The local field changes induced by these eddy currents will have different impact on the image depending on the direction of the diffusion encoding gradient direction. This is illustrated in Figure $8 \mathrm{~b}$, where a diffusion gradient applied along the $x, y$, and $z$ direction causes the image to be sheared, scaled, or translated, respectively. This example is for illustration purposes only, the level of eddy current effects are normally about 2-4 $\mathrm{mm}$. This will cause each DWI to be hampered by a linear combination of these effects for a given measured diffusion direction. As the amount of shear, scaling, and translation is dependent on the diffusion direction, ${ }^{35}$ and because the DTI data are calculated from all these DWIs, this implies an anatomical incon- 


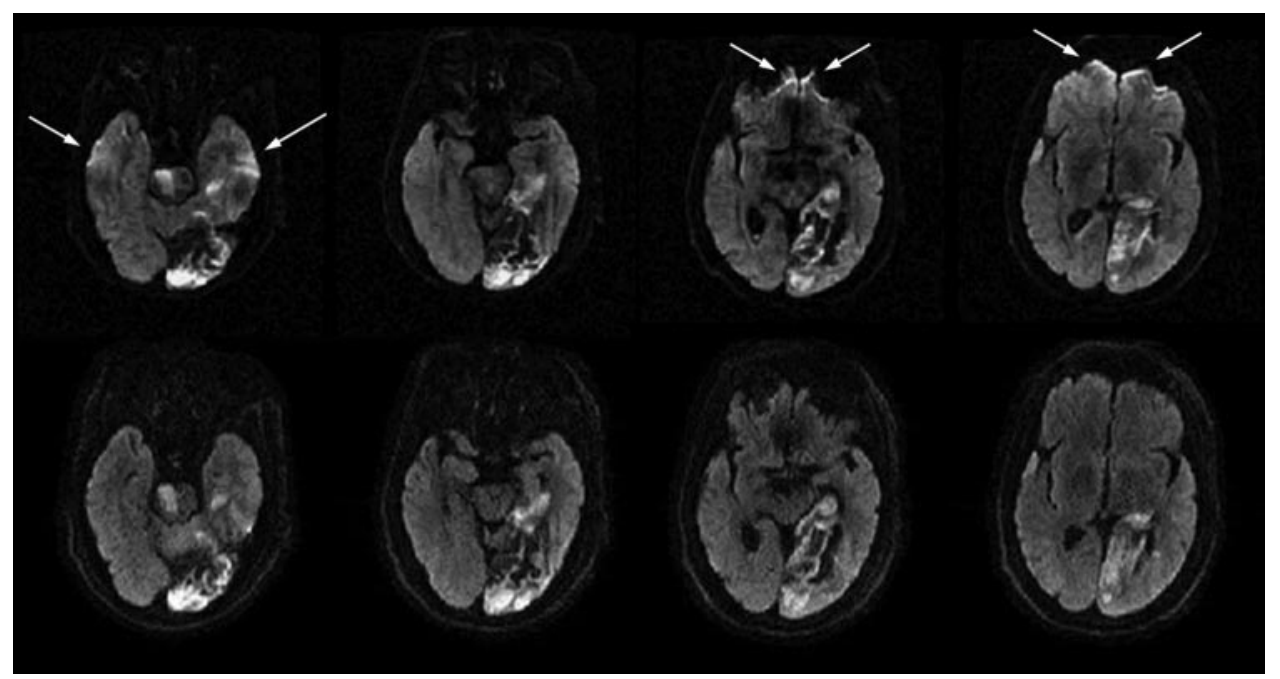

FIG. 9. Comparison between conventional diffusion-weighted single-shot EPI (a) and SENSE-EPI readout (b) in a stroke patient. By means of the faster $k$-space traversal with SENSE, the magnetic susceptibility artifacts or artifacts from $B_{0}$-inhomogneities can be markedly diminished.

sistency in the acquired data in addition to the possible geometric distortions from the true object shape. To obtain an anatomically consistent DTI data set and derivations thereof $(<\mathrm{D}>$, RA, etc.), the eddy currents need to be minimized (FIG. 3c). Here, four diffusion gradients are applied, and two $180^{\circ}$ pulses are used. ${ }^{36}$ In this way, the majority of the eddy currents are negated before the EPI data acquisition. In addition, postprocessing solutions exist that correct these image distortions on the basis of image coregistration. ${ }^{33,37-39}$

With the recent introduction of parallel imaging, the quality of EPI scans in terms of reduced blurring and geometric distortions caused by off-resonant spins can be significantly improved ${ }^{30,31}$ (FIG. 9). The accelerated $k$ space traversal is directly related to the reduction factor in parallel imaging. In addition to conventional gradient encoding to form an image, parallel imaging capitalizes on the distinct sensitivities of individual elements of receiver coil arrays. The additional spatial information provided by these coils allows one to sample $k$-space more sparsely and, hence, more quickly.

In addition to the comparatively standardized singleshot EPI imaging techniques, there are other promising ways to acquire the DWI data. Some of the most promising techniques will be discussed in the further sections.

Radial scanning. Radial, or projection reconstruction, imaging is the original method by which MRI data were acquired. Here, radial lines or spokes are acquired that intersect an arbitrary axis in $k$-space at varying degrees (FIG. 10a). Radial acquisitions have the benefit of repeatedly sampling the center of $k$-space, ${ }^{40}$ which can act as a built-in navigator. Translational motion will introduce a constant phase offset and linear phase ramp on the data. These offsets are usually uncorrelated across lines of data. The radial acquisition contains the center of $k$-space in each line. This gives a common point of reference, from which a constant phase offset may be determined and removed. ${ }^{41}$ While a linear phase ramp may also be detected across the radial lines, it is common practice to simply convert to the projection domain (i.e., the Fourier transform of the observed echo), and perform a magnitude operation on the complex projections (i.e., discarding any residual phase). ${ }^{42}$ This has the effect of centering the projection and, therefore, removing the translation. Rotational motion will shift the effective $k$ space location. ${ }^{41}$ Should the rotation shift the $k$-space location along the current radial direction, the effect is a simple one dimensional translation of the line, which can be recentered. However, should the rotation shift the effective $k$-space away from the radial line, the data measured will no longer be a radial line. These data are spurious and, although detectable, cannot be corrected. Radial acquisitions, however, are immune to most motion errors, and those remaining artifacts are more benign than would be seen with a Cartesian acquisition (FIG. 10b).

Recently, the periodically rotated overlapping parallel lines (PROPELLER) ${ }^{43}$ technique has been introduced as a novel method that incorporates both two-dimensional (2-D)-navigation information with the benefits of a radial acquisition. This multishot technique covers a thin strip or blade of $k$-space in each acquisition (FIG. 11a). By controlling the refocusing pulses between reading lines of $k$-space, phase information between lines is preserved. Furthermore, the inherent oversampling near the origin of $k$-space allows a number of phase conditions to be evaluated. Many conditions may also be corrected. However, if the data contain uncorrectable errors, such as a $k$-space shift from a rotation large enough to move the center of $k$-space outside the sampling of the blade, these 

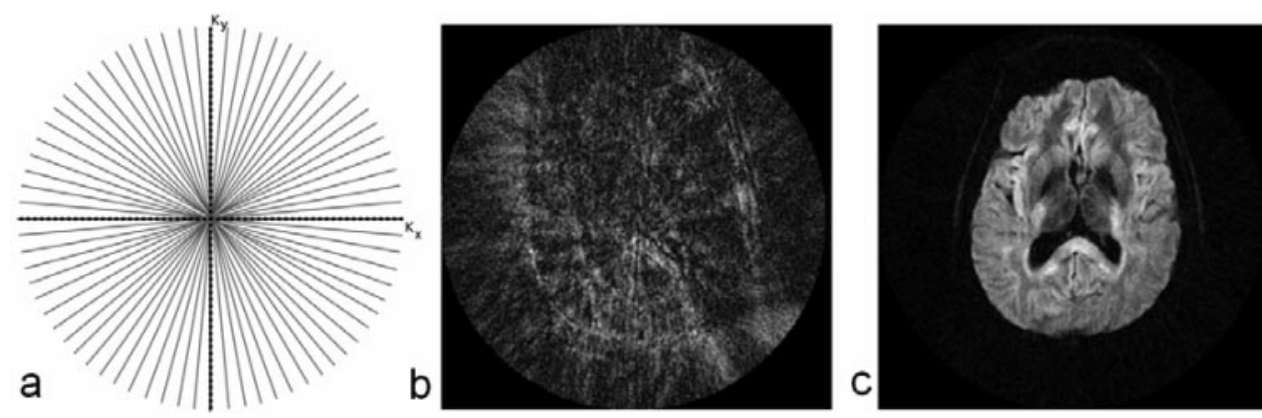

FIG. 10. a: A radial or projection reconstruction acquisition, lines of $k$-space are acquired at varying angles, each of which passes through the center of $k$-space. b: Without echo centering the distortions of each projection are quite significant and lead to mediocre image quality. c: High-resolution DWI scan (same as in panel b) after echo centering and magnitude-only reconstruction.

data may be detected and removed from the final data set. PROPELLER DWI has been demonstrated to be useful in areas with severe susceptibility distortions where EPI suffers from strong image degradation, such as areas close the base of the skull or in association with metallic material $^{44}$ (FIG. 11, b and c).

Spiral imaging. A few studies have recently explored the self-navigating capability of the spiral readout trajectory in multishot DWI. ${ }^{45,46}$ Previously, MRI based on spiral readout has been found to be effective in applications such as functional neuroimaging ${ }^{47,48}$ and spectroscopy. ${ }^{49}$ The spiral trajectory has the merit of momentnulling motion compensation and efficient use of gradient power. However, conventional spiral readout trajectories have limited potential for self-navigation because of the lack of data in the central $k$-space. This effectively allows only zero- and (to some extent) firstorder compensation. To improve the navigating capability, it is necessary to increase the sampling density at the center of the $k$-space. With a recently developed analytic variable-density (VD) spiral design technique, ${ }^{50}$ the $k$ space sampling density can be easily increased around the origin of $k$-space. This leads to a diffusion-weighted multishot VD spiral sequence design, ${ }^{51}$ a self-navigated technique and promising alternative for high-resolution DWI. With the analytic VD spiral design, the $k$-space sampling density can be easily manipulated and prescribed on the scanner hardware in real time. Here, each spiral oversamples the central region of $k$-space and undersamples the outer region of $k$-space. The sampling density is circularly symmetric and decreases smoothly as the radius increases in $k$-space. This trajectory is rotated consecutively in several repetition times to achieve full coverage of $k$-space. To reduce the eddy currents caused by the switching of diffusion gradients, VD spiral can be combined with a twice-refocused spin echo (TRSE) sequence. ${ }^{36,52}$ The image reconstruction involves two main steps: gridding ${ }^{25}$ and phase correction. ${ }^{26}$ The gridding step resamples the $k$-space data onto regular Cartesian grids to use the fast Fourier transform. For each spiral, the phase error in image space is then corrected by a low-resolution phase map estimated from the center portion of the $k$-space. The final image is formed by a direct combination of images acquired by each spiral. The image quality is improved dramatically with VD spiral and can further reduce the signal drop-off caused by motion-induced phase error. Early examples of high-quality fractional anistrophy maps with in-plane resolution of $0.86 \times 0.86 \mathrm{~mm}^{2}$ are shown in Figure 12 .

\section{Diffusion MRI-based tract tracing}

The ability to outline axonal fiber bundles in neuronal networks is important for the understanding of normal and pathological processes affecting brain function. For example, cognitive and motor processes that involve a variety of functional areas are mediated by such neural networks. In concert with fMRI, ${ }^{53}$ the availability of a noninvasive technique that outlines fascicles could enhance the understanding of the spatiotemporal interaction of normal brain function and adaptive processes such as brain plasticity. Finally, DTI may play an important role in treatment planning of neurosurgery or dose sculpting in radiation therapy by adding this technique to the diagnostic battery available to the oncologist.

Thus far, tract tracing of the human brain has been solely applied to postmortem studies. These methods include the dissection of white matter, ${ }^{54-56}$ strychnine neuronography, ${ }^{57}$ and neuronal degeneration associated with localized lesions. ${ }^{58-60}$ Histologic methods are based on active axonal transport of tracer molecules and have been applied mostly in animal studies. Unfortunately, such passive diffusive tracer studies of the postmortem brain also require several months to trace very short distances and are affected by cross-fiber diffusion ${ }^{61}$ which impairs spatial distinctiveness. Therefore, a noninvasive, reproducible, and repeatable tract tracing method is of greatest importance.

Fortunately, the directional information afforded by the anisotropic diffusion characteristics in white matter provides exactly this framework for tracking. For example, if one assumes that, in each voxel, the orientation of 

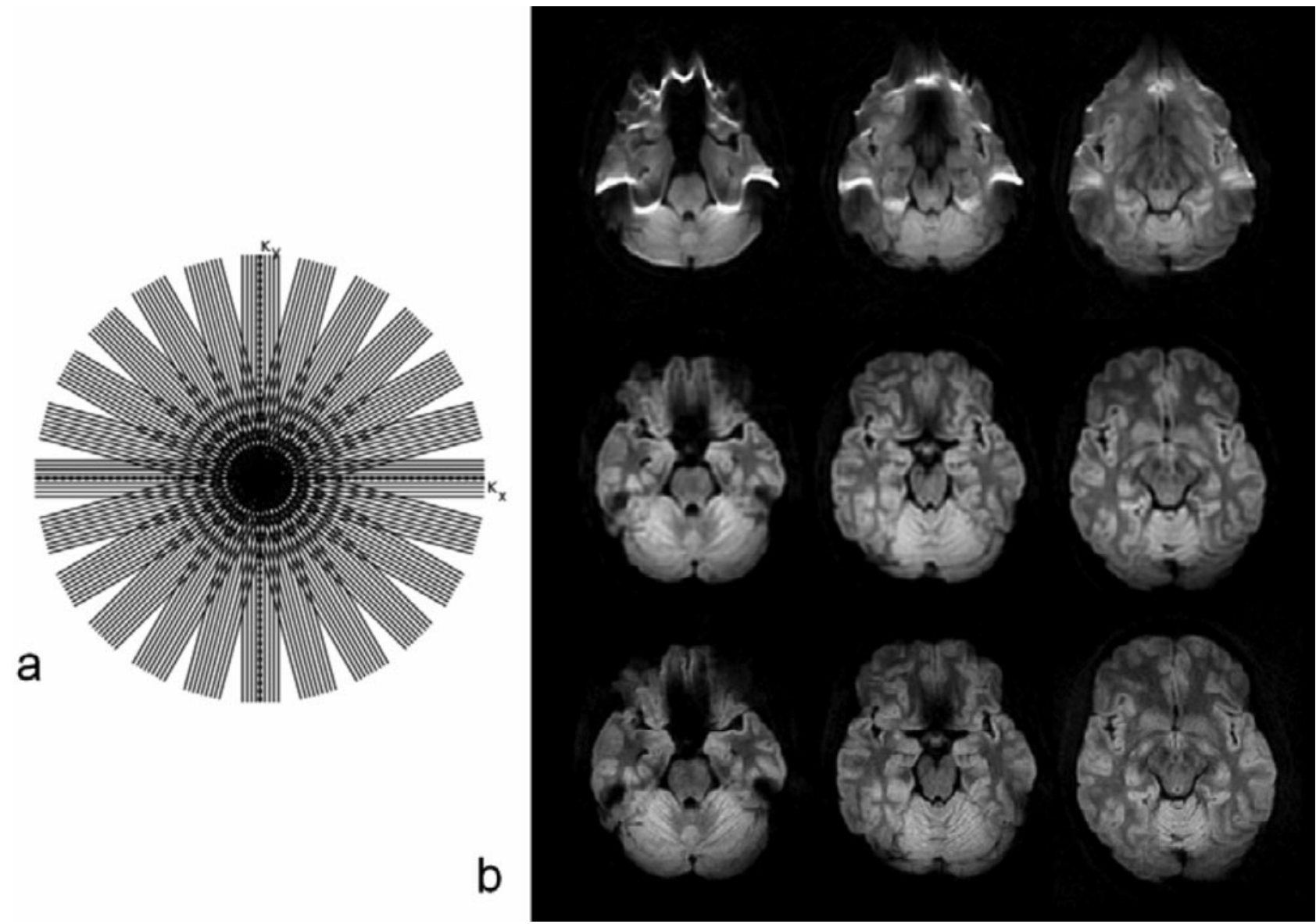

FIG. 11. a: A PROPELLER trajectory blade uses a Cartesian set of lines, with a greatly reduced $k_{\max }$ in one direction. This set of lines is then rotated about the origin of $k$-space, and acquired again. This leads to a set of intermediate images which are at the desired spatial resolution along the readout direction but have significantly lower resolution along the shorter dimension of the blade. These intermediate images allow one to perform phase navigation and leads to markedly improved image quality. b: DWI at 3T: Top row: EPI DWI demonstrates significant artifacts near air tissue interfaces. Typical signal pile up and loss as well as strong geometric distortions are apparent. Middle row: PROPELLER DWI with identical acquisition matrix demonstrates very few artifacts and provides significantly better image quality. In this implementation of PROPELLER, five gradient echoes were acquired between each refocusing RF pulse to speed up the acquisition. The overall acquisition time was comparable to the EPI scan. Bottom row: PROPELLER DWI at twice the acquisition matrix demonstrates high level of detail without significant distortions.

the eigenvector associated with the largest eigenvalue is aligned with the predominant underlying fiber orientation, tractography can be achieved by connecting each voxel to the adjacent one. This is performed by following the direction toward which the local direction vector points. However, stepping from one voxel to another is limited to the choice of only eight neighboring voxels ${ }^{62}$ in 2-D (or 26 in 3-D), and so the tracking often deviates from the true fiber orientation. Propagating from one voxel to the next nearest neighbor may result in nonsmooth trajectories which are most likely erroneous. To avoid the "granularity" of discrete space, a continuous representation of the vector field can be used and an individual fiber tract can be computed (FIG. 13a). ${ }^{62-64}$ In the continuous domain, the endpoint is not required to be the closest grid position, and this allows a more accurate tracking. At the endpoint, the direction of preferred diffusion is calculated again and, for a small increment, the tract propagates further along the newly found direction. Mathematically, this is simply a numeric integration operation. This step can be iterated many times until certain stopping criteria are met. For instance, to ensure that the traces would not erroneously pass into areas of very low anisotropy, such as CSF or gray matter, the tracking process can be stopped when a diffusion anisotropy measure (e.g., fractional anisotropy) falls below a fixed threshold (usually ranging from $0.1-0.15$ ). In addition, it has been proposed that only a limited amount of curvature per spatial increment (e.g., $10^{\circ}$ /increment) should be tolerated between subsequent steps. ${ }^{65}$ Tracking results are only sound if 1) the tensor information truly reflects the orientation of the underlying fibers; and 2) the numeric integration is accurate.

Individual tracking algorithms vary predominantly by way of orientational information, i.e., whether only the first eigenvector or the whole tensor is interpolated/ap- 


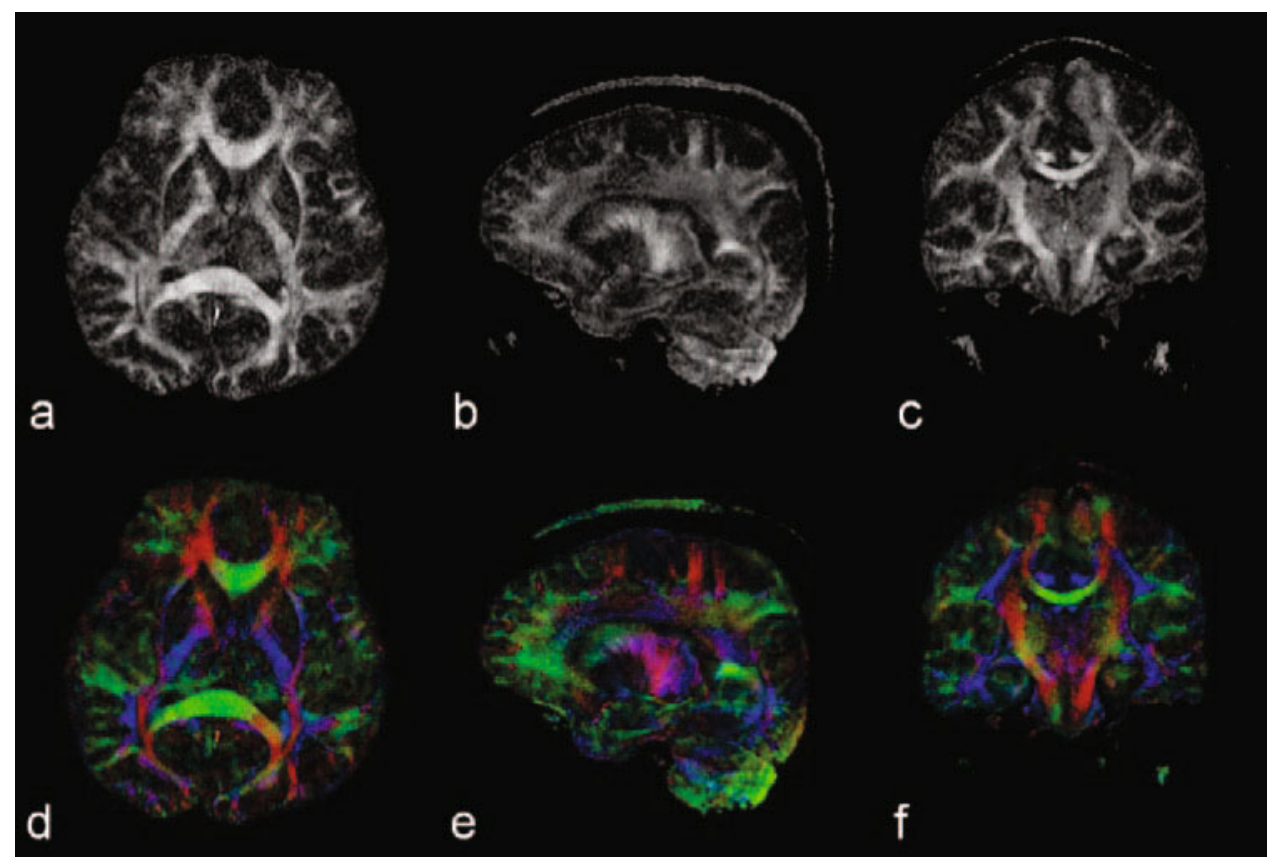

FIG. 12. Top row: In vivo high-resolution $(256 \times 256)$ DTI results acquired with a VD spiral TRSE sequence. a-c: FA maps with axial, sagittal, and coronal orientation. Bottom row (d-f): Color-coded FA map. Red represents the direction of anterior-posterior; green represents left-right; and blue represents cephalo-caudal.

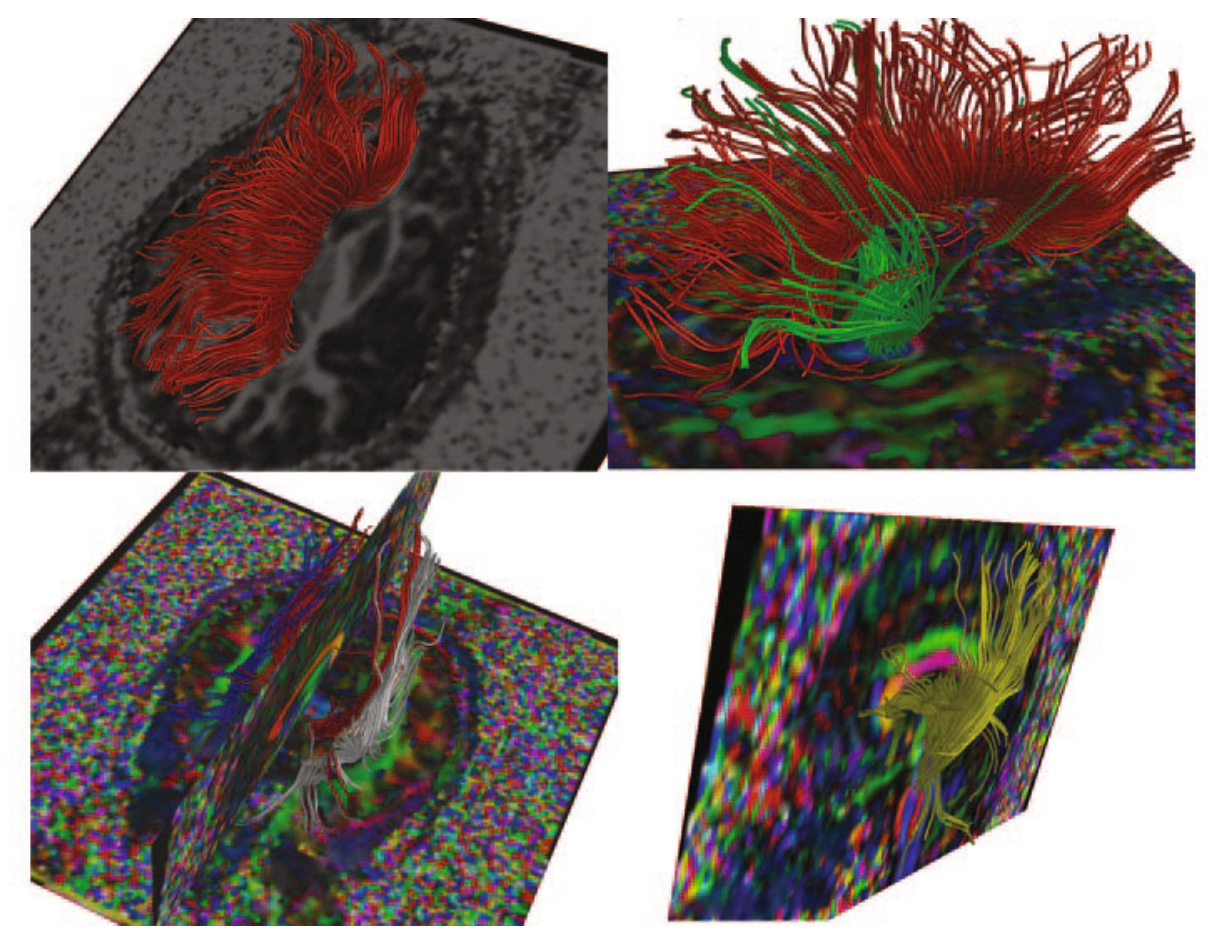

FIG. 13. Fiber tracking results from a software built in house. 3D renderings of different fiber tracts are superimposed on axial and sagitta FA maps. Fiber orientation on FA maps is highlighted by color coding (left-right $=$ red; anterior-posterior $=$ green; cranio-caudal $=$ blue). 


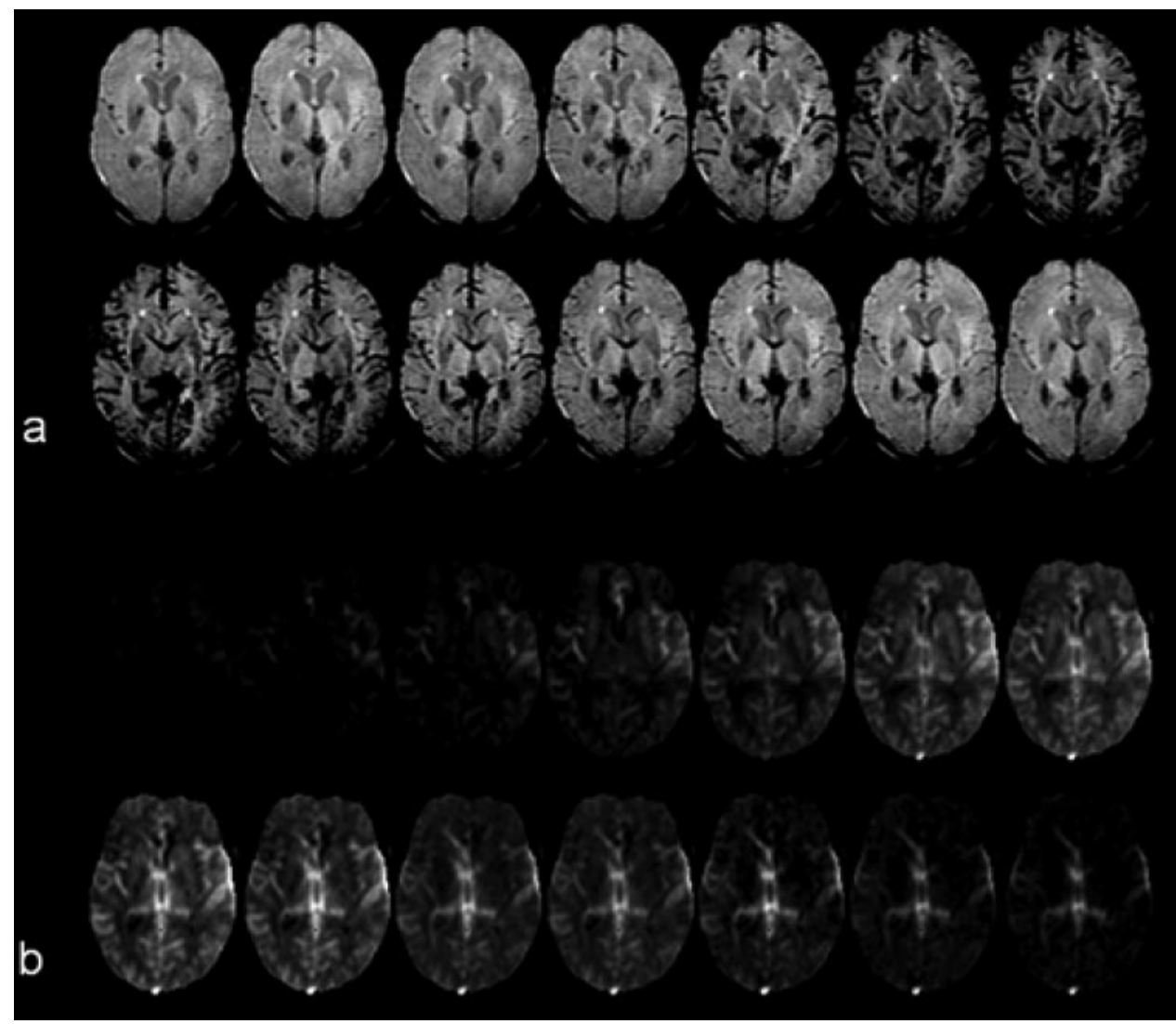

FIG. 14. Dynamic susceptibility contrast imaging. a: Time course of the $\mathrm{T}^{*}$-weighted $\mathrm{MR}$ images during contrast material bolus passage. Due to the high concentration of contrast material the signal intensity decreases significantly during the peak of the bolus. b: The corresponding concentration time course demonstrates highest signal intensities during the peak of the bolus passage.

proximated. In addition to point-to-point tracking methods, probabilistic tracing methods were recently derived (FIG. 13b). ${ }^{10,66-68}$

\section{PERFUSION}

Perfusion-weighted MRI (PWI) is an evolving MRI technology for studying cerebral hemodynamics and blood flow. ${ }^{69,70}$ Hemodynamic maps of cerebral blood flow (CBF), cerebral blood volume (CBV), and mean transit time (MTT) or time to bolus peak (TTP) can be created. These maps are based on mathematical analysis of the evolution of the intensity of the $\mathrm{T} 2 \%$-weighted gradient or spin echo images after a gadolinium bolus administration or by using "labeled" water protons as endogenous, freely diffusible tracers. The advantages of these PWI techniques are their high resolution and noninvasive nature compared with PET- or CT-based methods. PWI can be combined with other MRI techniques such as magnetic resonance angiography (MRA) to assess vessel patency and DWI to assess ischemic injury. ${ }^{6}$

\section{Measuring perfusion with MR: basic theory}

Two techniques are currently available to measure cerebral perfusion with MR: 1) dynamic susceptibility con- trast based techniques (also called bolus-tracking techniques) that use paramagnetic contrast agents; and 2) arterial spin labeling techniques that use magnetically labeled blood water as an endogenous tracer.

Dynamic susceptibility contrast-based techniques. Unlike CT or PET tracers, ${ }^{71}$ MRI contrast agents and their concentration in tissue/blood cannot be measured in absolute concentrations but rather in relation to their interaction with the hydrogen protons in their neighborhood. In addition to changes in longitudinal and transverse relaxation rates, paramagnetic contrast agents like gadolinium or dysprosium chelates generate susceptibility changes in the local magnetic field when administered to peripheral blood. These local susceptibility gradients reduce the $\mathrm{T} 2 *$ relaxation time and cause a loss of signal intensity when the contrast agent passes through the vasculature and capillaries. As the contrast disappears, the susceptibility changes decrease, and both the magnetic field inhomogeneity and the signal loss are reduced. Usually, heavily $\mathrm{T} 2 / \mathrm{T} 2 *$-weighted gradient echo or spin echo techniques are used. Spin echo techniques are more sensitive to microvascular perfusion but require more contrast agent to produce a similar signal to noise ratio in the temporal intensity response curve. To capture the fast bolus passage (usually on the order of a few seconds) and 

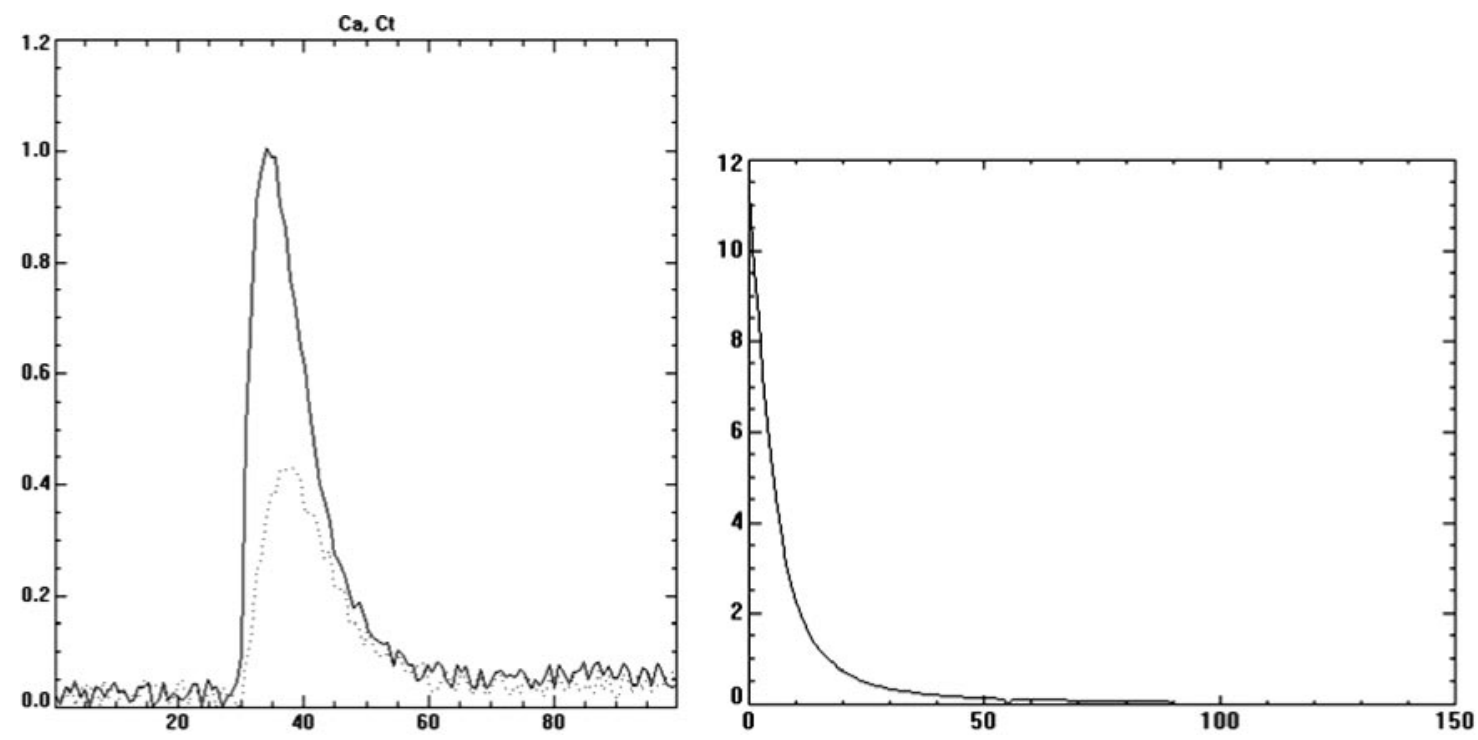

FIG. 15. a: Contrast agent concentration time course in the feeding artery ( $C_{a}$; AIF: solid line) and corresponding tissue response signal $\left(C_{t}\right)$. b: Tissue residue function obtained by deconvolving $C_{t}$ from $C_{a}$.

measure the susceptibility changes, rapid imaging methods like EPI are required (FIG. 14). The amount of signal loss induced by the contrast agent changes exponentially with increasing concentration of contrast and is proportional to the $\mathrm{CBV} .{ }^{72-75}$

For every pixel in the brain, a curve can be created that depicts the changes in signal intensity over time as contrast enters and exits the brain. The formula depicting these changes is:

$$
\Delta \mathrm{R} 2 *(\mathrm{t})=\Delta(1 / \mathrm{T} 2 *) \propto-\ln \left(\mathrm{S}(\mathrm{t}) / \mathrm{S}_{0}\right) / \mathrm{TE}
$$

where $S_{0}$ is the precontrast signal intensity, $S(t)$ is the contrast signal intensity at time $t$, and $T E$ is the echo time. Here, $S_{0}$ is usually the mean of several baseline images (10 or more) that are averaged after the signal has reached its steady state (usually one to four images). To minimize error propagation due to noise, a larger number of baseline images $(>20)$ is advisable to reduce noise in the denominator in the equation above.

Given the proportionality of the signal change over time with contrast concentration, a curve depicting the contrast concentration over time can easily be constructed (FIG. 15). The area under this curve is proportional to the CBV in healthy brain tissue where it is assumed that the blood-brain barrier is unaffected and no contrast extravasation occurs. This assumption also holds true early after onset of cerebral ischemia where vasogenic edema due to blood-brain barrier disruption is delayed. ${ }^{76,77}$ In tumors or subacute stroke where extravasation is likely, it has been suggested to "preload" the patient with some gadolinium contrast to allow extravasation, thereby minimizing the concentration gradient when the true dynamic study is conducted.
Summary parameters. A simple approach to obtaining information about the hemodynamic status is to analyze the time intensity curve using time parameters calculated directly from the profile of this curve. Commonly used parameters are: 1) TTP, 2) bolus arrival time, 3) maximum peak concentration, and 4) full width at half maximum, etc. Sometimes, maps of 1) CBF, 2) CBV, and 3) MTT are provided, by applying the central volume principle to these curves. The central volume principle relates MTT (the normalized first moment of the efflux concentration after injection of an indicator) as the ratio of the $\mathrm{CBV}$ with the tissue blood flow. ${ }^{78}$ Because $\mathrm{CBV}$ can be obtained by calculating the area under the curve and the MTT is measurable from the curve as the first moment of the concentration-time curve, CBF maps can be obtained as well. However, the transit times that are measured with MRI are unreliable because no real efflux transit times are evaluated but rather the amounts of contrast that remain within the tissue, i.e., a residue distribution. ${ }^{79,80}$ No simple relationship exists between the transit time distribution and the residue distribution to measure flow without knowledge of the underlying vascular structure. These simple methods also disregard variations in injection conditions of the contrast bolus (e.g., injection rate and duration) and patient physiology (e.g., changes in cardiac output), making intersubject or within subject comparisons difficult. Still, some authors have found that these methods worked equally well than the more complex methods described below. ${ }^{81}$

Deconvolution approaches. When an idealized bolus of contrast (i.e., infinitely short and high) is injected into a tissue with quantity $q_{0}$, the concentration of contrast within a region of interest at a time $t$ and, hence, the 


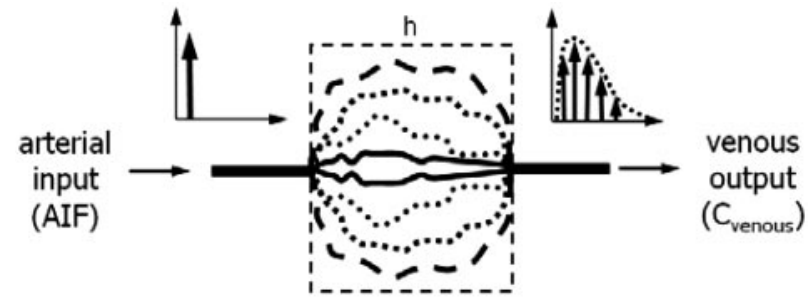

FIG. 16. Capillary model of transit times. Due to varying capillary retention times, parts of the instantaneous bolus take longer to travel through the capillary network than others. For such an instantaneous bolus (i.e., infinite height, infinitesimal short) the venous output reflects the distribution of transit times and is equivalent to the impulse response function of the capillary system. The transit time distribution allows the computation of the mean transit time. The shape of a nonideal bolus affects the venous output.

obtained signal $S(t)$ is proportional to: 1) the ratio of blood flow within the tissue relative to the flow in the artery that feeds the tissue; 2) the fraction of contrast remaining within the tissue at times $(t)$, known as the residue function; and 3) the amount of contrast itself. The residue function is by definition $R(t)=1-h(t)$, where $h(t)$ is the fraction that has left the voxel of interest (VOI) at time $t$ and, therefore, $R$ at time 0 equals 1 . The function $h(t)$ is the impulse response function of the capillary system or the probability density function for the contrast agent transit times through the VOI (FIG. 16)

This formula implies that blood flow can be determined if the residue function is calculated from the arterial input function into the VOI and the signal intensity curve within the VOI. Here, the initial response of an impulse bolus is proportional to blood flow because by definition, $R=1$ at $t=0$.

Mathematically expressed, the contrast in the tissue of interest $\left(C_{v o i}\right)$ is proportional to blood flow (CBF), a proportionality constant dependent on brain density and hematocrit within plasma $(\kappa)$, and to the convolution of the arterial input function $(A I F)$ with the residue function $R$. This is:

$$
\mathrm{C}_{v o i}=\kappa \cdot \mathrm{CBF} \cdot[\mathrm{AIF} * \mathrm{R}(\mathrm{t})]
$$

where the second function AIF is the arterial input function, which is the concentration of contrast agent in the feeding vessel to the region of interest at time $t$ and * describes the convolution operation. A real bolus of contrast is of finite duration, therefore a correction is required as if multiple, idealized boluses are given to obtain the residue curve. A correction for this noninstantaneous bolus can be provided by performing a deconvolution.

The deconvolution can be performed using several mathematical techniques that are outside the scope of this work. The most commonly used technique is the model independent truncated singular vascular decom-

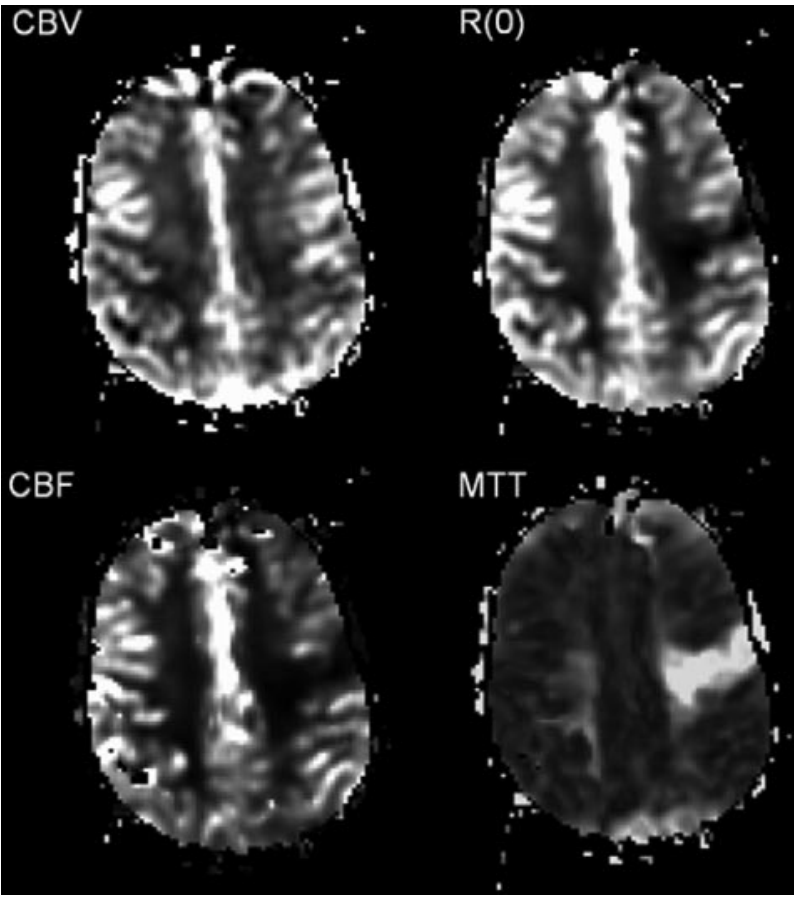

FIG. 17. Acute stroke patient. CBV, CBF, MTT and R(O) map of the residue function at $t=0$ computed with the TSVD deconvolution approach. Clear perfusion deficits in the left middle cerebral artery territory are apparent.

position method (TSVD) described by Ostergaard and colleagues. $^{82,83}$

The CBV can be measured by calculating the area under the measured concentration curve and normalizing it to the integrated arterial input curve, i.e., the arterial blood volume. By applying the central volume principle, the MTT can be measured as the ratio of CBV to CBF. Compared with the aforementioned summary parameters this provides a much "cleaner" estimation of the CBF and MTT (FIG. 17). Several studies have compared CBF values from PWI with PET or Xenon CT and found strong correlations in normal, healthy volunteers. ${ }^{82,84-86}$

Several problems remain, however, regarding the use of PWI to noninvasively quantify CBF and MTT in pathological states. The problems relate to the difficulty of measuring brain density and plasma hematocrit in pathological states and obtaining a value for the relaxivity of gadolinium contrast agent across a range of blood vessel sizes. Other pitfalls include the difficulty of measuring the arterial input function close to the voxel of interest, delay and dispersion of the contrast bolus. ${ }^{87}$

Arterial spin labeling. Cerebral perfusion measurements can also be achieved using the arterial spin labeling (ASL) approach, ${ }^{88,89}$ which utilizes magnetically labeled arterial blood water as a diffusible tracer for CBF measurements. A similar principle is used for $\mathrm{H}_{2}{ }^{15} \mathrm{O}$ PET scanning. ${ }^{71}$

With some exceptions, current methods and recent developments are based on the original ASL method first 

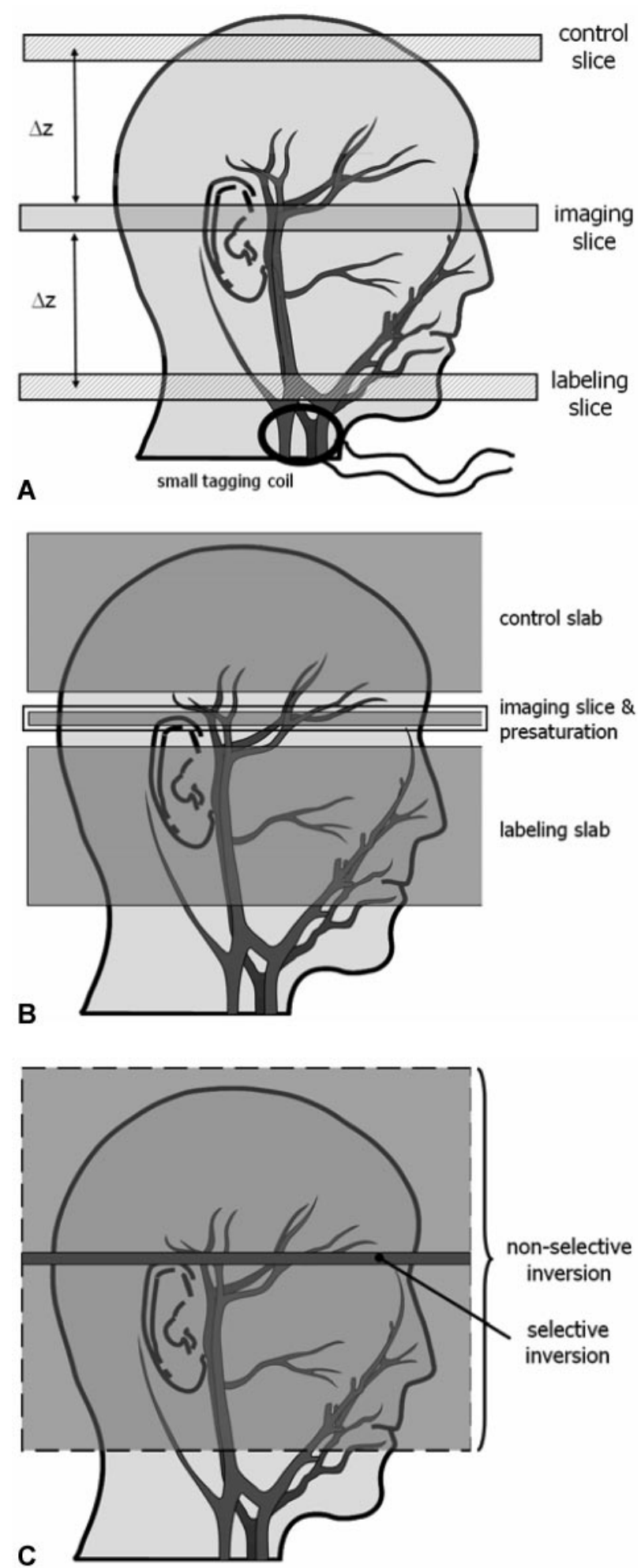

FIG. 18. A: Continuous arterial spin labeling of inflow in blood in the labeling slice leads to a slightly altered $\mathrm{T} 1$ relaxation in the measurement slice. Magnetization transfer can partially saturate spins in the measurement slice and may falsify perfusion measurements. A control experiment with labeling at the same distance distal to the measurement slice can counterbalance the MT effect. Venous return can be an additional confounder. Small local label coil are another alternative to restrict the MT

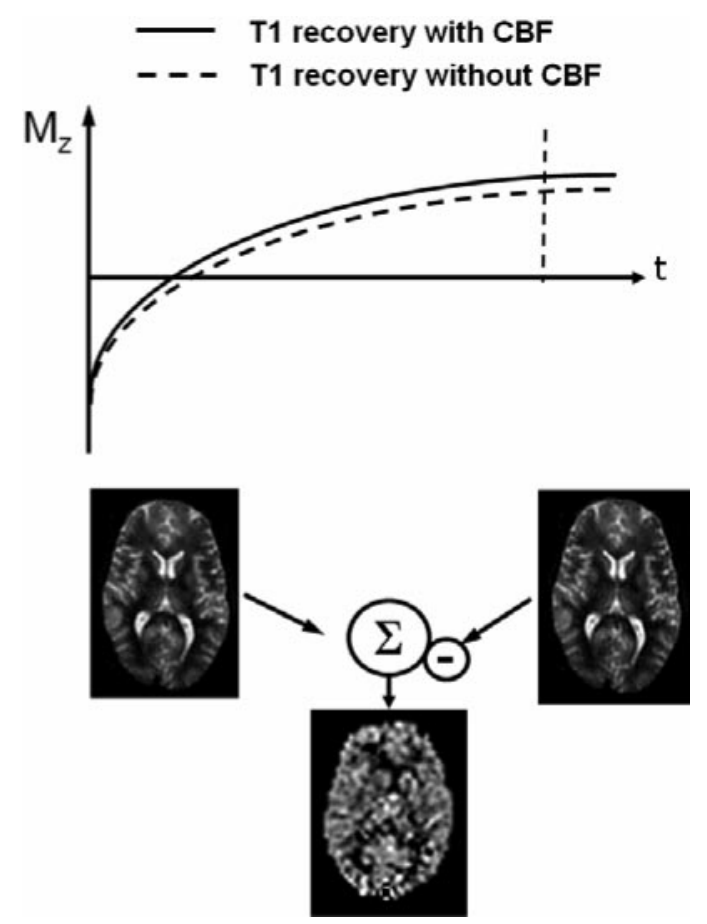

FIG. 19. T1 recovery is dependent on cerebral blood flow. Subtracting the labeled from the control acquisition results in a difference image which provides qualitative information about cerebral perfusion.

proposed by Williams and Detre et al.$^{88,90}$ These authors employed a continuous adiabatic inversion technique to invert the flowing spins in the carotid arteries (FIG. 18a). The influx of fresh, labeled protons in the tissue of interest slightly alters the magnetization and, depending on the exchange with tissue protons, the $\mathrm{T} 1$ relaxation time of the tissue. This method is sensitive to the local degree of microperfusion. ${ }^{88}$ Hence, any change in regional blood flow will be picked up by ASL via image contrast changes (FIG. 19). The effect of spin labeling in blood will decay with the relaxation time of $\mathrm{T} 1$. This signal decay is generally considered to be sufficiently slow to allow the labeled blood to travel from the labeling site to the microvasculature and tissue of interest. The aforementioned ASL approach is often referred to as continuous ASL (CASL). This may generally suffer from in-

effect and avoid venous return artifacts. Moreover, these coils allow selective assessment of vascular territories. B: EPISTAR and PICORE are typical representatives for pulsed ASL (PASL). With EPISTAR after saturation of the imaging slice, a slab proximal to the imaging slice is labeled using a single, short RF inversion pulse. The blood in this slab is then allowed to flow into the imaging slice, and an image is acquired after a time TI. A control image is also acquired for which the label is applied distal to the imaging slab (EPISTAR) or with no slab selective gradient (PICORE). C: Two inversion recovery images are acquired. The first follows a slice-selective inversion and thus has a signal intensity determined by an apparent, inflow-modulated T1 time; the second follows a global inversion and so (assuming blood and tissue relax at the same rate) has no flow enhancement (i.e., the image signal intensity is determined by intrinsic tissue $T_{1}$ ). 
sufficient spin inversion and magnetization transfer (MT) effects that are induced by the adiabatic pulse with a slice selection gradient. MT occurs between water protons and protons bound to macromolecules via chemical exchange and dipolar coupling. Because the bound protons exhibit a much broader line shape they become unintentionally saturated by the inversion (labeling) pulse that erroneously results in a pseudo-perfusion effect in the measurement slice. The MT effect can be counterbalanced by a simple control labeling experiment where the labeling is done at the same distance from the measurement slice at a distal location. Because the MT effect depends on the slice position, more sophisticated control experiments are required in a multislice ASL measurement, such as the use of a separate labeling coil with limited B1 penetration or a sinusoidal modulation of the radio frequency (RF) wave form. ${ }^{91}$

The introduction of pulsed ASL (PASL) methods has undoubtedly contributed to the widespread use and interest in ASL mainly due to practical considerations. The EPISTAR sequence was the first PASL method and was proposed by Edelman et al. ${ }^{92}$ Before or after an inversion pulse in a proximal, widened slab an additional saturation of static spins in the volume of interest is performed to eliminate possible contamination of the inversion slab (FIG. 18b). Similar to the original CASL sequence, a second experiment with distal inversion is needed to control for MT effects. Based on the EPISTAR sequence, several variations and improvements have been proposed. Most of them focus on a better control of MT effects in a multislice experiment, such as the transfer insensitive labeling technique (TILT), ${ }^{93,94}$ the proximal inversion with control for off-resonance effects (PICORE) sequence ${ }^{95}$ or the double inversion with proximal labeling of both tagged and control images (DIPLOMA) sequence. ${ }^{96}$

A second class of PASL methods relies on symmetric labeling with respect to the imaging volume. In the simplest case, this is achieved with an inversion recovery sequence that is performed twice (with and without slice selective inversion). This is known as flow-sensitive alternating inversion recovery (FAIR) ${ }^{97}$ (FIG. 18c). In the case of slice-selective inversion, all the spins in the vessels outside of the imaging volume are labeled in the sense that their magnetization differs from the magnetization of blood in the imaging volume. The difference resulting from these two acquisitions yields a perfusionweighted image. MT is usually a minor problem in PASL compared with CASL methods due to the reduced RF power needed and the fact that the MT effect is counterbalanced over the center slice. There are several further developments with acronyms indicating that they are based on the FAIR sequence. The UNFAIR ${ }^{98}$ and FAIR$\mathrm{ER}^{99}$ sequences are performed with additional RF pulses to null static signal in the volume of interest, whereas the
FAIREST $^{100}$ sequence separately determines $T_{1}$. If the T1 relaxation time at both labeling conditions is known, ASL techniques are capable of quantifying CBF (FIG. 20). Quantitative ASL models are based on the extended Bloch equation for the longitudinal magnetization. The extended Bloch equation includes additional exchange terms for influx and efflux magnetization. This implies that labeled blood and tissue magnetization can be considered as a single well-mixed compartment and that mixing occurs instantaneously when the arterial blood enters the voxel or volume of interest. In addition to restricted magnetization exchange between the intravascular and extravascular compartment, quantitative errors may also result from the labeled blood itself. Insufficient labeling or inversion as well as variations in the transit time may contribute to such errors. This poses a serious problem in patients who have stenotic vessels or in deep white matter in which the mean blood transit time is relatively long. Additionally, a problem arises from the remaining labeled signal in the vasculature that is often reflected by high perfusion values after image subtraction. This is particularly a problem for single-shot readouts with low spatial resolution. Generally, ASL can be combined with all imaging readout principles. However, in many cases one resorts to fast spiral or EPI readouts, especially for fMRI ${ }^{101,102}$ or when quantitative perfusion maps are warranted. Alternatively, fast spin echo sequences and interleaved spirals can be used for moderately fast scanning with much better image quality. ASL is well known as a low signal method. The perfusioninduced change of tissue magnetization is usually on the order of a few percent of the steady state magnetization. An advantage is expected from higher field strength where the benefit for ASL methods is twofold: first, the higher baseline SNR; and second, the longer T1 relaxation time of blood. Here, a longer T1 time implies that a tagging pulse persists longer and, therefore, is of advantage for ASL.

Considering all current capabilities and restrictions, ASL can provide reliable perfusion maps of a large portion of the brain. However, current ASL methods are often complex and still susceptible to motion and other problems. This is probably also the reason why ASL still has not replaced nuclear medicine methods although the latter are usually more invasive and do not provide a better resolution. Nevertheless, ASL has been successfully applied to investigate perfusion abnormalities in patients with stroke and other vascular diseases. ${ }^{103}$ However, this is to a much lesser extent than perfusion studies based on dynamic susceptibility contrast. ASL has been also applied in fMRI studies. fMRI is an MRI technique that allows a direct mapping of functionally active areas (see Functional MRI). Functional activation can be observed by an increase in regional blood flow. ASL has proven extremely valuable for fMRI because it does not 

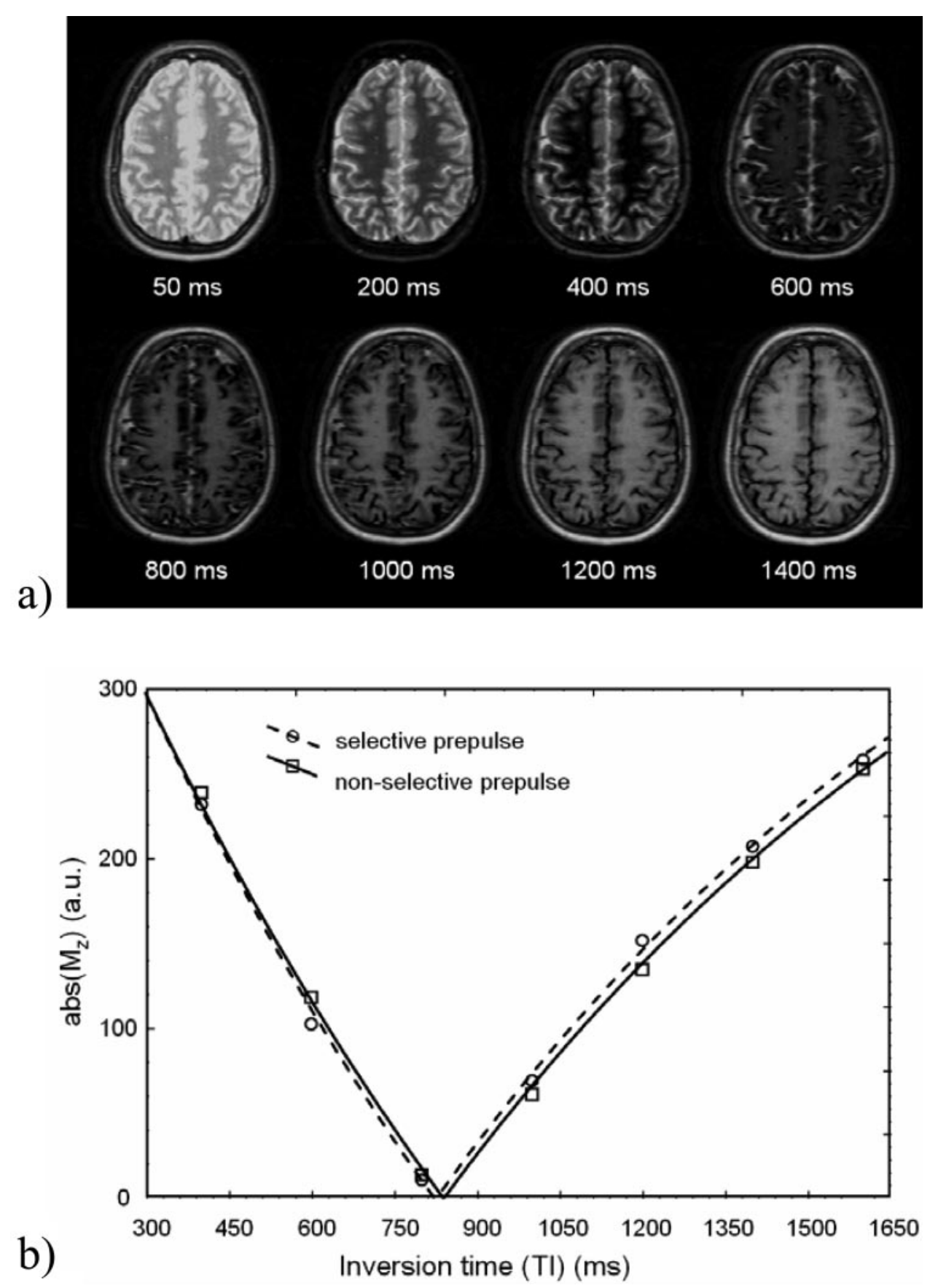

FIG. 20. FAIR ASL experiment with variable inversion times. a: Resulting IR-FSE images obtained with different inversion times. b: Course of signal intensities depending on TI. Spins from the selectively inverted experiment experience a slightly faster T1 recovery due to the inflow of unsaturated blood. The IR curves allow the calculation of T1 maps from which, together with knowledge of the brain partition coefficient, a quantitative perfusion map can be computed.

require the administration of contrast material and can be repeated multiple times.

\section{DWI and PWI and the Delineation of the Ischemic} Penumbra in Ischemic Stroke: The Mismatch Hypothesis

A variety of MRI signal profiles can be seen in acute stroke patients. The most common MRI profile in pa- tients with acute stroke is a PWI lesion that is larger than the DWI lesion (FIG. 21). ${ }^{6}$ Some patients have an isolated PWI lesion without any DWI abnormality. Approximately $70 \%$ of patients imaged within the first $6 \mathrm{~h}$ after stroke onset will demonstrate this PWI/DWI mismatch. ${ }^{104-107}$ The next most common MRI pattern encountered in acute stroke patients is an early DWI lesion that is larger than the PWI lesion (or no PWI lesion is 


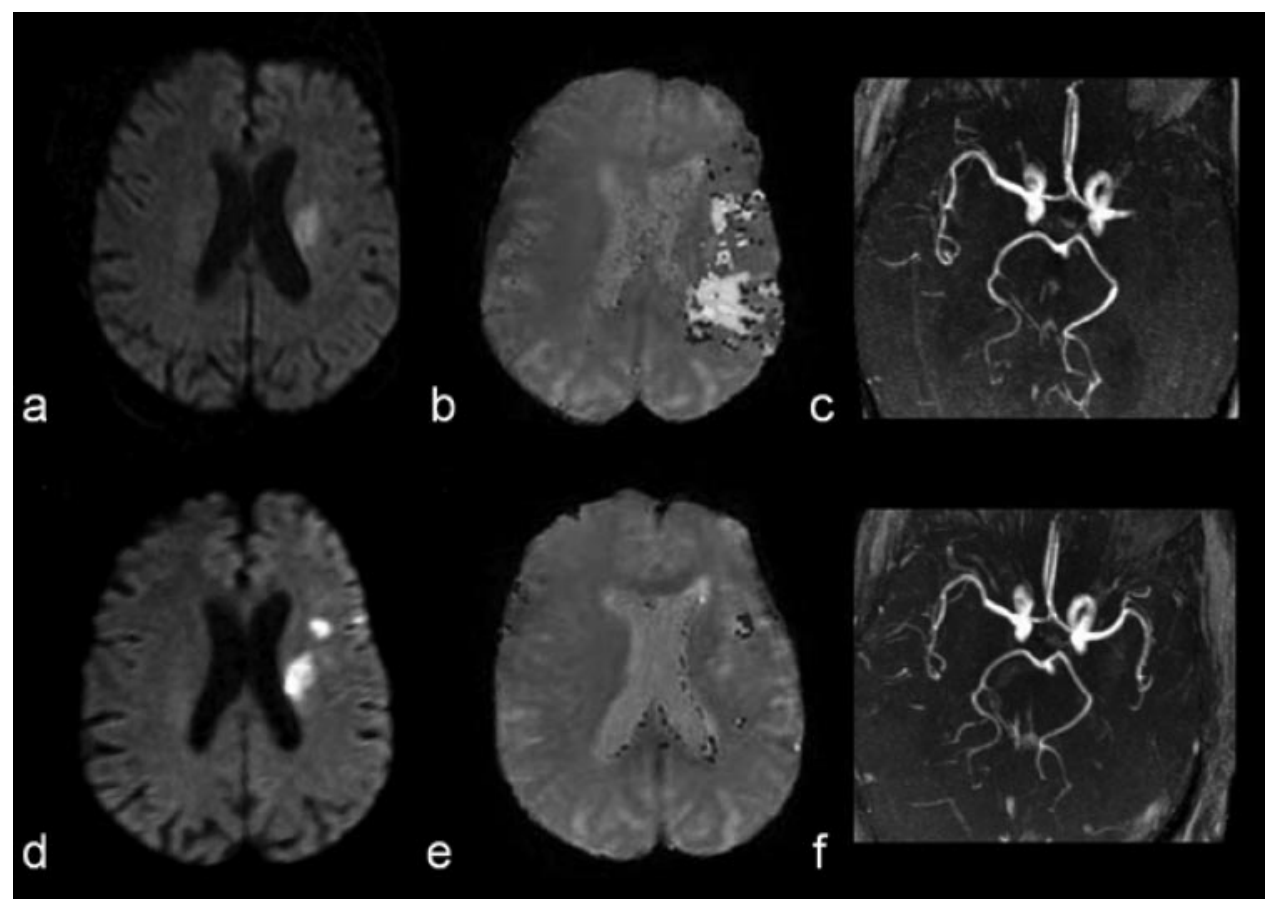

FIG. 21. Acute stroke patient with successful recanalization after IV tPA treatment. a: DWI within $6 \mathrm{~h}$ after stroke onset demonstrates a small lesion in the left middle cerebral artery territory. b: The area of perfusion deficit is apparent from the TMAX image (overlay on T2*-weighted baseline scan) and confirmed by complete occlusion of the MCA in the time of flight MRA (c). d-f: Follow-up scans $6 \mathrm{~h}$ later demonstrate successful reperfusion ( $d$ and $f$ ) and minimal lesion growth (d).

present). These patients are thought to have experienced partial or complete spontaneous recanalization. Rarely, no DWI or PWI lesion is seen on early imaging. This is usually seen in patients in whom spontaneous resolution of the neurologic deficit has occurred or in whom very small infarcts with limited clinical deficits are present. Several arguments suggest that the mismatch between the PWI and DWI lesion may be an estimate of the ischemic penumbra. Baseline DWI lesions may represent the ischemic core and the mismatch between the lesion identified on PWI and DWI may represent an area with critical hypoperfusion, the ischemic penumbra. Several arguments support this mismatch hypothesis:

Hypoperfusion lesion volumes correlate with clinical impairment scores better than acute DWI lesions. A high correlation has been found between the lesion volume identified on acute PWI with acute neurologic impairment scales in patients whose PWI lesion was larger than the DWI lesion volume. The correlation with DWI in this early time period was lower. This suggests that the PWI lesion represents functionally impaired tissue. ${ }^{108-110}$

The evolution of DWI lesions is dependent on the presence of hypoperfusion. In untreated stroke patients, DWI lesions typically enlarge during the first week after symptom onset. ${ }^{104-106,111-113}$ Perfusion lesions typically decrease in volume over time. ${ }^{105}$ In a randomized, placebo-controlled trial of the putative neuroprotective agent citicoline, MRI showed that untreated patients studied within $24 \mathrm{~h}$ after symptom onset had follow-up lesion volume $180 \%$ the size of the baseline lesion volume, although there was substantial variability. ${ }^{114} \mathrm{Fac}-$ tors that were independently associated with greater lesion growth were the presence of a large hypoperfusion lesion on PWI, vascular occlusion on MRA, and larger size of the baseline diffusion lesion. Patients studied within $12 \mathrm{~h}$ also had more substantial lesion growth. These findings confirm previous observations using DWI and PWI in acute stroke patients. ${ }^{105,112,113,115}$ For example, a serial study in 49 stroke patients performed imaging during the first day after symptom onset, at day 2 and at 1 week. Eighty percent of the patients had a PWI/DWI mismatch at baseline. The mismatch volumes correlated significantly with DWI lesion growth between day 1 and day 2 , and day 1 and 1 week $^{113}$

The PWI/DWI mismatch is time dependent. Darby et al. ${ }^{116}$ found a PWI/DWI mismatch in $75 \%$ of stroke patients scanned within $6 \mathrm{~h}$ of symptom onset, $70 \%$ at $12 \mathrm{~h}$, and $44 \%$ at $18 \mathrm{~h}$ after symptom onset.

Patients with a PWI/DWI mismatch who experience reperfusion have less lesion growth and better functional outcome compared to patients without reperfusion. Chalela et al. ${ }^{117}$ serially studied 42 patients with MRI before and 2-3 $\mathrm{h}$ after treatment with intravenous (IV) thrombolysis with recombinant tissue plamsminogen activator (tPA). The most powerful predictor for excellent functional outcome was the reduction of the volume of hypoperfusion by more than $30 \%$ between baseline and follow-up. In a pilot trial, Par- 
sons et al. ${ }^{118}$ serially studied 19 patients within the $0-6 \mathrm{~h}$ before IV tPA and at subacute and chronic time points. Patients who were treated with IV thrombolysis had more frequent reperfusion and recanalization and had improved clinical outcomes than a historical control group. ${ }^{118}$

Reversal of DWI lesions is infrequently found. Serial studies show that most DWI lesions develop into infarcts as defined by imaging or pathologic examination. ${ }^{104,119}$ However, there are also several limitations impacting on the mismatch hypothesis:

Areas within the PWI/DWI mismatch are not always at risk of infarction. In patients with a mismatch the DWI lesion typically grows over the first days after symptom onset to achieve a size that includes a large part of the initial perfusion deficits. ${ }^{107,112}$ Typically the initial early PWI lesion overestimates the final infarct volume and the DWI lesion does not grow to the full size of the initial perfusion lesion. ${ }^{81,108,120}$ The presence of tissue with minimal hypoperfusion or oligemia has been invoked to explain this phenomenon. Large hypoperfusion lesions can be found in patients without clinical deficit or in patients who do not develop infarctions. Typically, this occurs in patients with chronic large vessel occlusion where collaterals provide beneficial, but delayed perfusion that is difficult to detect with PWI. ${ }^{121}$

Some DWI and ADC lesions are reversible. Animal ischemia experiments have demonstrated that the lesion identified on DWI is larger than the area where ATP depletion has occurred, indicating that only part of the DWI lesion represents the ischemic core. ${ }^{122,123}$ Studies using apparent diffusion coefficient (ADC) mapping to provide a quantitative estimate of the degree of cytotoxic edema show that lesions with mild ADC can normalize, whereas lesions with more severe ADC reduction evolve into infarctions. ${ }^{124,125}$ Interestingly, ADC lesions can disappear entirely after early successful reperfusion, but reappear later. ${ }^{126,127}$ This delayed re-emergence of ADC lesions together with T2 and DWI hyperintensities occurs following prolonged hypoperfusion and is associated with the presence of tissue necrosis on pathologic examination. This resolution and re-emergence of DWI lesions is not associated with neurologic improvement followed by worsening. ${ }^{128}$

Permanent reversal of early DWI lesions has also been demonstrated in humans following early reperfusion confirming that early DWI lesions do not represent the ischemic core in stroke patients. ${ }^{129-133}$ Kidwell et al. ${ }^{134}$ showed the rapid resolution of diffusion lesions among patients with TIAs. The same group demonstrated that after recanalization with intra-arterial (IA) tPA therapy or combined IV/IA tPA treatment, some DWI lesion volumes regressed from baseline to follow-up. ${ }^{128}$ In $50 \%$ of these patients, this reversal of DWI lesions was tem- porary with the development of recurrent lesions within 1 week. This fluctuation of diffusion lesions appears very similar to the animal observations discussed above. Again, this pattern appears not to be associated with early clinical improvement and subsequent neurologic deterioration. ${ }^{135,136}$

Accurate determination of the core might be achieved by a quantitative analysis of the ADC map, together with parameters derived from PWI. Preliminary multiparametric analyses suggest that very low ADC values represent irreversibly damaged tissue and are also at higher risk of hemorrhagic transformation. ${ }^{135,137}$ Similarly, a quantitative analysis of the PWI lesion might be able to distinguish oligemia from critical hypoperfusion with PWI.

\section{FUNCTIONAL MRI}

One of the most exciting modern developments in MRI has been the noninvasive visualization of human brain function. Previously, the exclusive domain of the technology of positron emission tomography (PET), an important subset of MRI is now capable of mapping functional regions of the human cortex in real time during specific task activation. fMRI is now in use for the localization of visual, motor, and somatosensory responses in surgery of tumors, ${ }^{138}$ localization of "handedness," and elucidation of brain function and metabolism altered by pathologies such as stroke, multiple sclerosis, ${ }^{8}$ and Alzheimer's disease. ${ }^{139}$ Given the large number of clinical MRI scanners operating worldwide, aside from fundamental new understanding in neuroscience, fMRI is used in clinical assessment of brain function.

fMRI is achieved using inherent magnetic susceptibility image contrast characteristics of the iron in blood hemoglobin. Blood is therefore used as a local endogenous indicator of functional activation. This is because blood in its oxygenated state, i.e., oxyhemoglobin $\left(\mathrm{HbO}_{2}\right)$, is diamagnetic and causes small magnetic susceptibility changes without changing tissue $\mathrm{T} 2 *$, whereas deoxyhemoglobin $(\mathrm{Hb})$ has paramagnetic properties (due to four unpaired electrons) and perturbs the local magnetic field leading to an observable $\mathrm{T} 2 *$ shortening. This contrast mechanism is referred to as blood oxygenation level-dependent (BOLD) contrast. ${ }^{53}$

Localized brain activity is associated with variations in regional tissue oxygenation (due to changes in oxygen uptake and altered blood supply) can thereby be mapped by T2*-weighted MRI. fMRI has the exciting potential for high-speed, noninvasive, and high-resolution exams that can track functional changes as they occur in near real-time. Biofeedback can be used by the subject to alter perception (e.g., pain), or effect other behavioral 

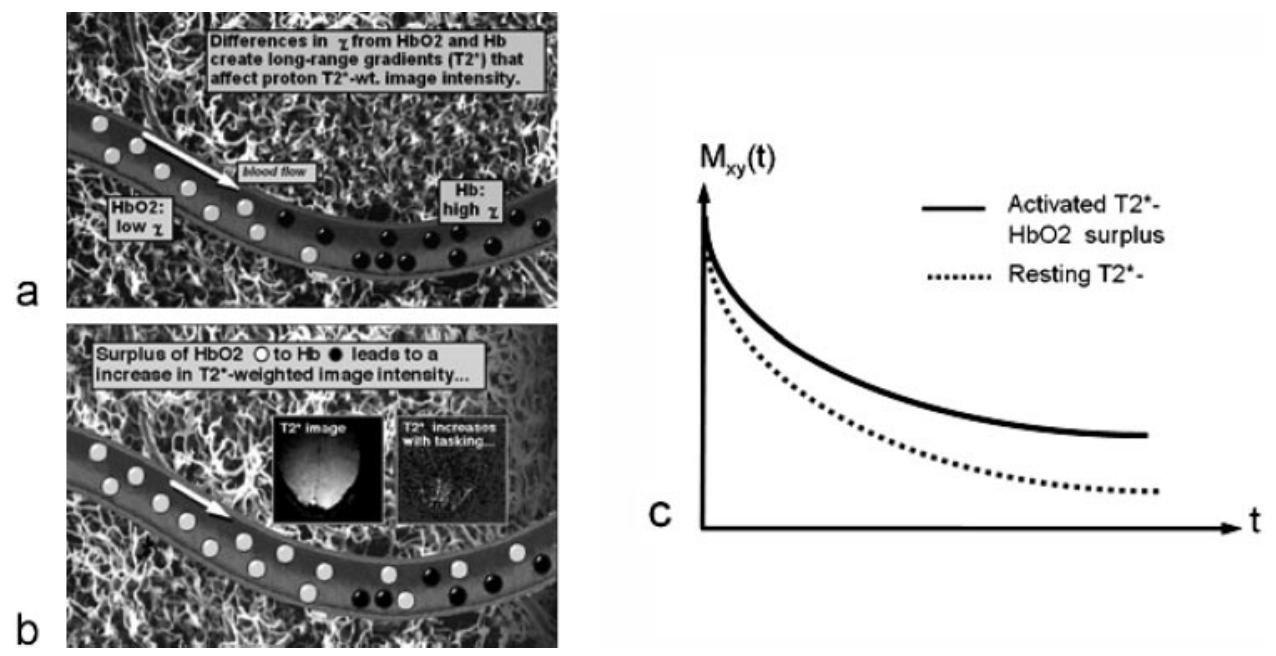

FIG. 22. a: Hemoglobin as an MRI contrast agent. Regional blood oxygenation or deoxygenation in the brain can result from changes in local or global metabolic-related oxygen uptake, and changes in blood flow. This is manifested as an increase or decrease in signal intensity in T2*-weighted images. As the oxygenation state of hemoglobin changes to deoxyhemoglobin, the iron becomes paramagnetic and thus alters the local T2* by disturbing the local B0. This in essence causes decreases in T2*. b: As more oxygenated arterial blood is delivered to the voxel the $\mathrm{T}^{*}$ is then increased, causing an increase in signal. c: The T2* is higher in the activated state. The echo time with the optimal fMRI contrast is about equal to $\mathrm{T}^{*}$.

changes. This capability has already become a reality for many of the rapidly developing fMRI research centers worldwide. These centers have typically involved a number of collaborating groups from radiology, neurology, neurosurgery, psychiatry, psychology, and physics departments. After circumventing initial problems, it is anticipated that fMRI will continue to evolve over the next several years and will spread far beyond the boundaries from which it arose.

\section{Background on fMRI}

A significant fraction of the CBV can be found in the capillaries and venules of the brain and is therefore available for imaging brain activation. A stimulus (e.g., visual, vibration, motor activity) causes neuronal activity that increases $\mathrm{CBF}, \mathrm{CBV}$, and hence oxygen delivery. $\mathrm{CBF}$ rises more than $\mathrm{CBV}$. $\mathrm{HbO}_{2}$ quickly exceeds local metabolic needs due to the stimulus and causes a surplus of fully oxygenated blood in the activated voxel. Due to the surplus, the capillary and venous beds fill with a larger ratio of $\mathrm{HbO}_{2}$ to $\mathrm{Hb}$ compared to the brain at rest. This slightly increases the local $\mathrm{T} 2 *$ relaxation times and, hence, increases the image intensity by a few percent (FIG. 22). Hence, magnetic susceptibility-induced $\mathrm{T} 2 *$ signal modulation reflects the local oxyenation state of the venous system. In addition, perfusion and diffusion of spins in the capillary bed through changing magnetic susceptibility has a direct impact on the observed T2 relaxation times, although the resulting signal changes are smaller than those from the susceptibility effect (especially at $1.5 \mathrm{~T}$ or lower field strengths).

The actual CBV is quite small (a few percent of the total volume); however, the $\mathrm{T} 2 *$ effects extend beyond the vascular bed.

A local $\mathrm{B} 0$ gradient can exist across the vascular tree from a diamagnetic $\mathrm{HbO}_{2}$-rich environment (with a longer relative $\mathrm{T} 2 *$ ) to a more "paramagnetic" $\mathrm{Hb}$ environment with a shorter $\mathrm{T} 2 *$. The arterial blood consists mostly of $\mathrm{HbO}_{2}$; however, as it passes through the capillary bed, the local concentration of deoxyhemoglobin increases and often predominates. The net observable fMRI contrast depends on the balance of $\mathrm{Hb}$ to $\mathrm{HbO}_{2}$ and is therefore a function of arterial autoregulation or vasodilation. By either increased regional influx of $\mathrm{HbO}_{2}$ or reduced oxygen extraction, an increase in regional $\mathrm{T} 2 *$ and image intensity can be observed. This also induces a decrease of $\mathrm{Hb}$, i.e., an increase in venous blood oxygenation and a longer effective $\mathrm{T} 2 *$. Thus, the image intensity for a given voxel in the brain can significantly increase if more oxygenated blood enters this region and fills the venous bed. This model is based on the assumption that functional activation causes local vasodilation, which is not accompanied by a significant increase in oxidative metabolism.

It had been noted as early as 1982 that MRI could noninvasively detect differences in blood oxygenation by virtue of the paramagnetic properties of $\mathrm{Hb}^{140}$ Turner et al. ${ }^{141}$ showed in 1991 that T2*-sensitive MRI could image those changes noninvasively. Today, this idea has developed into a major advance for MRI and functional neuroimaging. ${ }^{53,142}$ In essence, MRI can detect hemodynamic responses to nearly any perturbation to the brain, such as hypoxia, hypercarbia, apnea, and most importantly, task activation. ${ }^{143-145}$ 


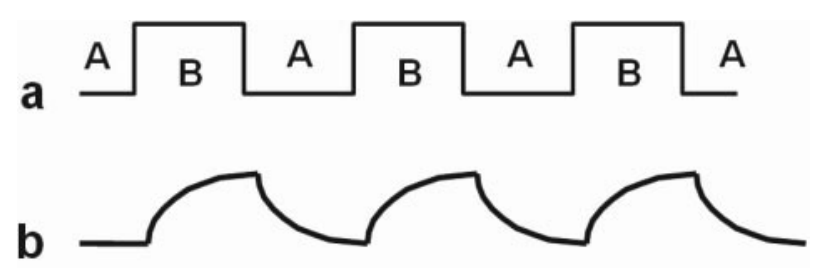

C

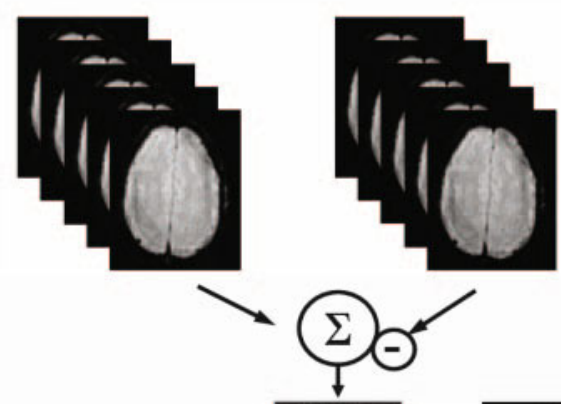

d

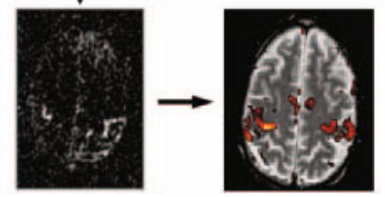

FIG. 23. $f M R I$ processing. a: $A$ usual $f M R I$ experiment is conducted by alternating between two states (A and B), e.g., stimulation ON/OFF. b: The tissue response follows the stimulation pattern determined by the hemodynamic response function. c: A combination of the images from states $A$ and $B$ leads to an image (d) that demonstrates contrast in areas where functional activation occurred. For better localization the activation results are overlaid in color onto a morphologic reference image.

Figure 23 demonstrates the general principle of fMRI contrast formation and postprocessing. The BOLD signal changes are small, typically only a few percent for activation in sensory regions, and even smaller for cognitive tasks. Therefore, it is customary to employ statistical processing methods to develop maps of activation from the time series of images generated during the scan. In such methods, either linear modeling or correlation algorithms are used to develop statistical parametric maps of the probability that voxel activity is related to the task. Many forms of such algorithms have evolved in attempts to eliminate both false positives (type I errors) and false negatives (type II errors). A number of free packages (e.g., SPM and AFNI) as well as commercial programs (e.g., BrainVoyager) are now available. Significantly, many MRI scanner vendors have recently introduced post-processing software, which should increase the availability and convenience of fMRI for clinical applications.

Figure 24 shows results from a typical finger apposition experiment, whereas Figure 25 demonstrates stimulation of the visual areas. Both experiments clearly demonstrate the superiority of increasing field strength (3T relative to $1.5 \mathrm{~T}$ ) for fMRI.

Although fMRI does not measure tissue perfusion directly, it can potentially contribute significantly to our understanding of organ metabolism by the quantification of oxygen utilization, as well as through tissue response to various therapeutic interventions. Because more than $70 \%$ of the brain's blood lies within the capillaries and venules, the measurement of magnetic susceptibility $\mathrm{T} 2 *$ signal loss predominantly reflects the regional deoxygenation state of the venous system. Under physiological conditions where diffusion does not limit the delivery of oxygen to the tissue, measurement of venous oxygenation will be critically linked to tissue oxygenation. The coupling of oxygenation-sensitive pulse sequences with high-speed imaging may ultimately become a powerful tool for assessing tissue oxygenation.

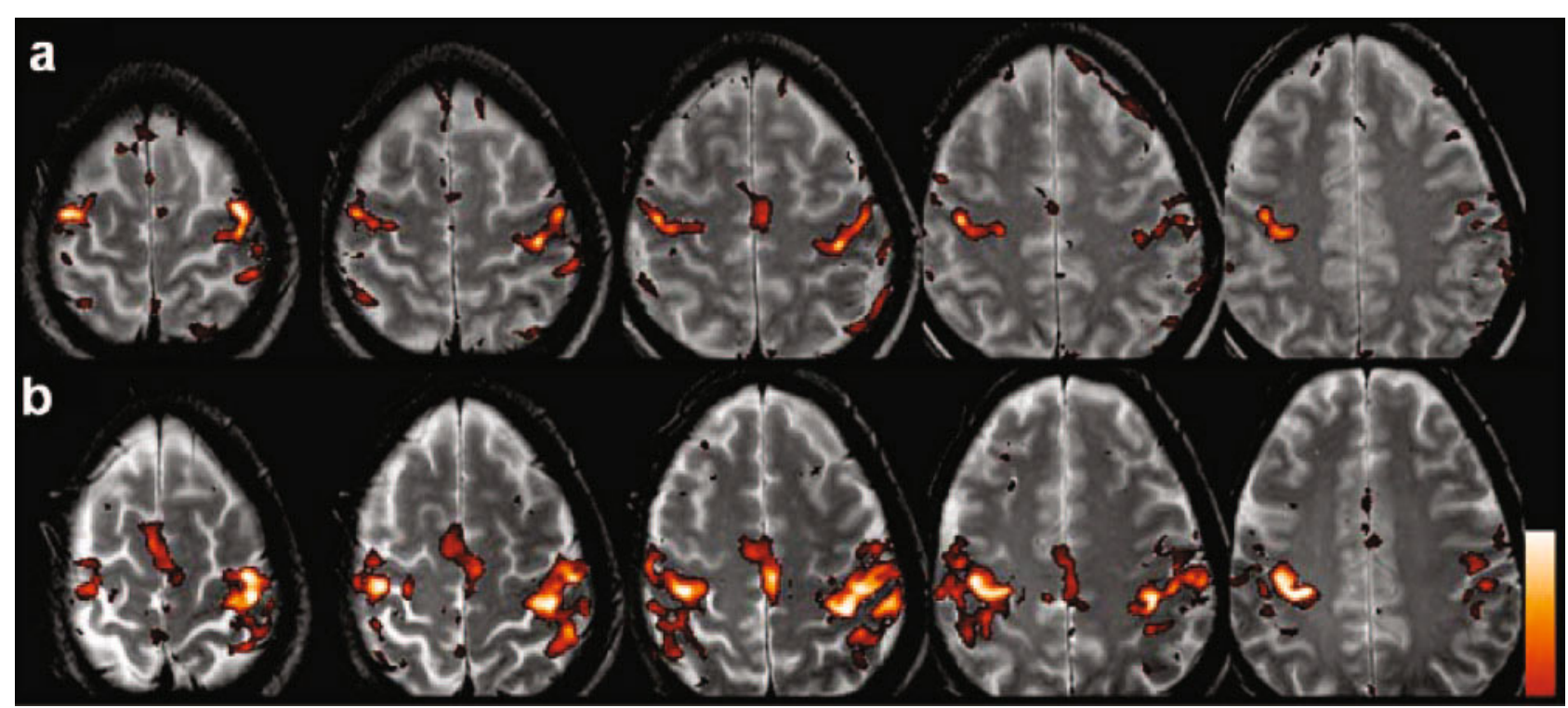

FIG. 24. fMRI experiment with finger apposition (both hands). The increased sensitivity to functional activation at $3 T$ (b) relative to $1.5 T$ (a) is apparent. 


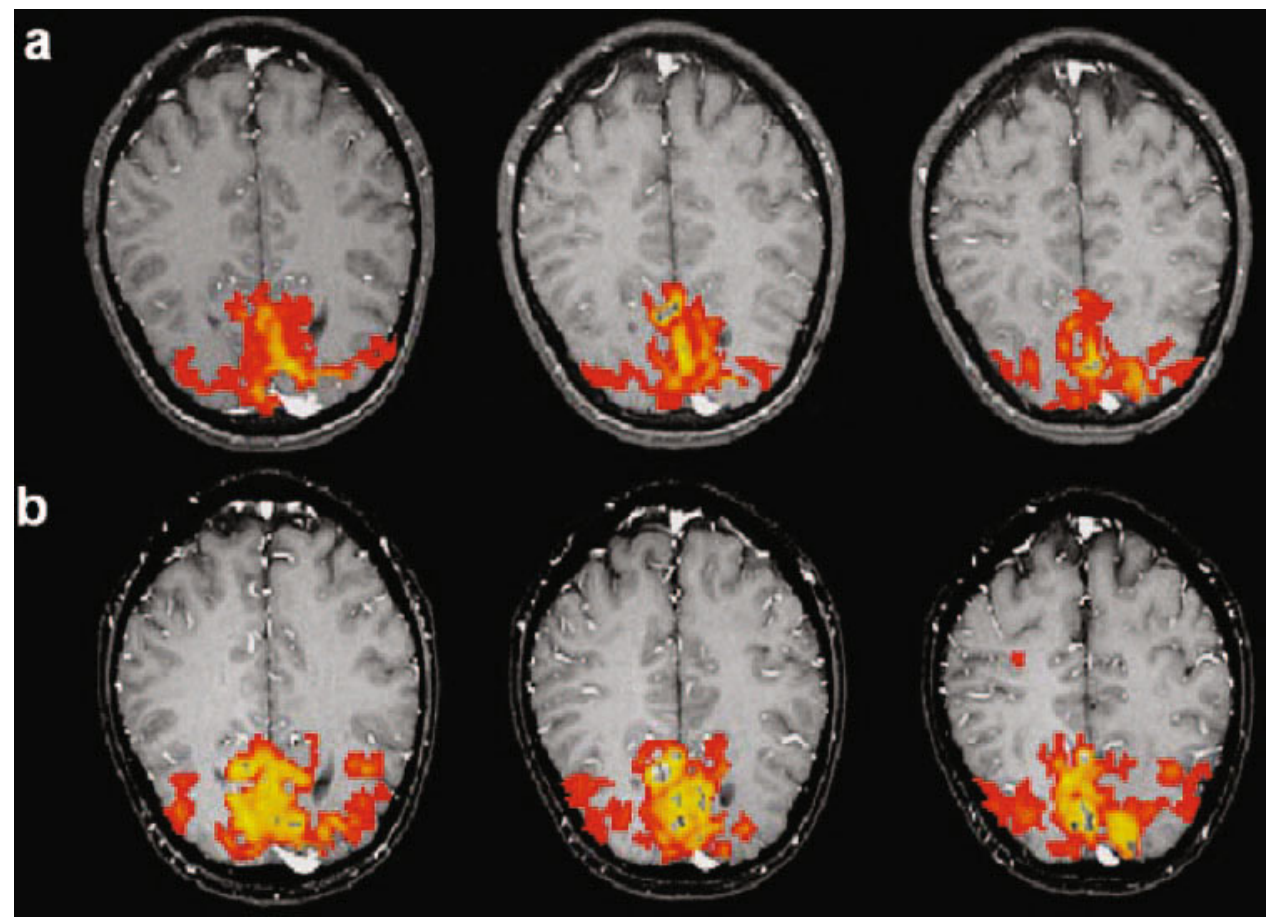

FIG. 25. fMRI experiment with finger apposition (both hands) (a and b) and visual stimulation (c and d). The increased sensitivity to functional activation at $3 \mathrm{~T}$ ( $\mathrm{b}$ and $\mathrm{d}$ ) relative to 1.5T ( $\mathrm{a}$ and $\mathrm{c}$ ) is apparent.

\section{A survey of task activation studies}

Functional MRI is promising with respect to the characterization of distinctly human functions as vision, hearing, language and memory. To understand brain function, the location of these processing sites must be identified, images of task performance within these sites acquired, and the organization of distributed processing determined. The observed physiological changes can be used to determine the functional loci of component mental operations.

Susceptibility contrast MRI techniques and a visual stimulus paradigm were first employed by Kwong et al. ${ }^{142}$ to produce these task activation maps. These maps were coregistered to high-resolution, T1-weighted, 3-D images of the underlying anatomy. This allowed investigators to accurately determine gray and white matter boundaries, as well as to delineate activated versus nonactivated borders. Functional and anatomical data sets were then translated into proportionately measured stereotactic coordinates relating to the line between the anterior and posterior commissures. This allowed the MRI maps to be directly correlated with reported, standardized PET maps of the same region. ${ }^{142}$ During photic stimulation of the volunteers $(n=7)$, each subject showed a significant increase in signal intensity within the anatomically defined V1 region, showing an average increase of $0.8 \%$ to $1.8 \%$. This demonstrated the temporal response of V1 signal intensity with both techniques. The results showed that hemodynamic alterations accompanying neuronal activation lead to subtle, but readily detectable, changes on $\mathrm{T} 2 *$-weighted echo planar images.

Bandettini et al. ${ }^{144}$ have described the dynamic characteristics of functional imaging using the effective time resolution of SE EPI. The rise time of the signal, which corresponds roughly to the increase in $\mathrm{CBF}$ in response to cortical activity, was on the order of a few seconds. Interestingly, a finger tapping task lasting only $0.5 \mathrm{~s}$ can produce a short-lived rise in signal with a delay of some $5 \mathrm{~s}$, so that the signal rises after the task is completed. The author later demonstrated postprocessing by crosscorrelation with reference vectors to create high-quality brain activation images and to extract spatially distributed phase differences in activation.

Various investigators have shown localized brain activity in response to a variety of tasks, such as silent word generation, visual mental imagery, and hand movements. Upon visual stimulation, reports observe a task-related signal change in the lateral geniculate nucleus. Others have described studies of visual and motor imagery in which subjects were asked to visualize the flashing red light of photic stimulator goggles, or to mentally rehearse a hand movement. In each case, areas of the brain close to or overlapping the primary region for that modality showed an increase in signal during the task.

Passive listening to speech sounds has been observed to induce more intense and widespread activation-induced signal change than white noise. Other studies demonstrating cerebral dominance and handedness, observed signal intensity changes in the left and right frontal lobes 
in response to word generation tasks, lateralized activation in Broca's area during internal speech, and activation in the parieto-occipital sulcus upon imagination of scenery. Activation is also seen with short-term memory recollection and in short-term sensitization.

Aside from capitalizing on the $\mathrm{T} 2 *$ changes during brain activation in fMRI, investigators have recently demonstrated that associated susceptibility changes and, hence, altered local off-resonance effects can also be detected from significant phase changes in balanced in steady-state free precession imaging. ${ }^{146}$ Several groups have now suggested that ADC also changes during brain activation. $^{147}$

Overall, it seems clear that the characterization of distinctly human functions, such as language and memory, as well as methods for assessing pathophysiology of the CNS, must be conducted by noninvasive, functional means. By application of fMRI, we are now able to obtain MRI maps of normal and pathologic mental operations. Whereas functional maps have previously been produced by radionuclide techniques (primarily PET), fMRI contributes a high-resolution in vivo, safe method. In conjunction with other functional measures, particularly PET, electroencephalography and magnetoencephalography, one is able to map the unique structure-function correlates involved in cognitive processing and is poised to make great contributions to our theoretical and philosophical formulations of mental activities. Potential clinical applications of fMRI include: 1) mapping of distortions of the functional motor and somatosensory cortex and other cortical regions as a result of brain tumors; ${ }^{138}$ 2) observing the effect of degenerative diseases such as MS, ${ }^{8}$ Alzheimer's disease, ${ }^{139}$ stroke and others causing neuronal loss; 3) Parkinsonism; and 4) the critical area of rapidly and noninvasively mapping normal and pathologic brain reactivity to any number of mild, reversible stresses.

\section{HIGH-FIELD MRI}

The current trend toward higher magnetic fields (3T and higher) is mainly driven by the expected gain in the signal-to-noise ratio (SNR) that roughly correlates with the field strength. The higher SNR may be used to: 1) obtain higher spatial resolution; 2) better contrast; and 3) to reduce MRI scanning time. Thus, systems operating at $3 \mathrm{~T}$ and higher have become an important adjunct in neuroimaging. The potential improvement in spatial resolution at $3 \mathrm{~T}$ and higher offers the opportunity to better visualize structural changes and to characterize signal properties of individual lesions. Thus, high-resolution imaging may potentially provide better insight into the complex pathophysiology of certain diseases and support an improved evaluation of patients. To that end, it can be anticipated that an increased number of smaller lesions

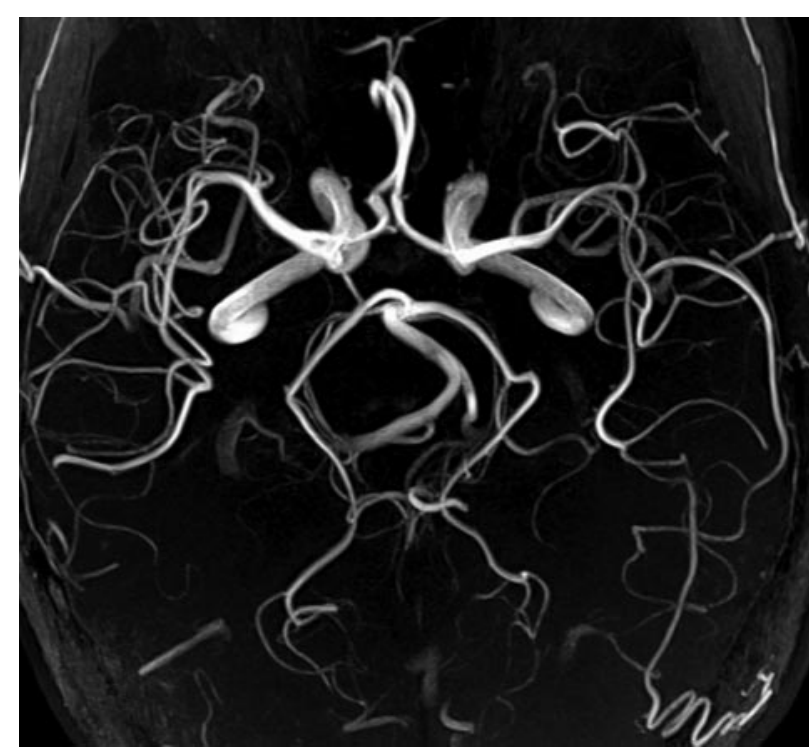

FIG. 26. MR-ToF maximum intensity projection (MIP) reconstruction demonstrating the strong benefit at $3 T$ from increased $\mathrm{T} 1$ relaxation times. Even finest vessel structures appear that are not observed at 1.5T. The strong signal at $3 \mathrm{~T}$ has been traded in high spatial resolution $\left(0.2 \times 0.2 \times 0.6 \mathrm{~mm}^{3}\right.$, 3T MAGNETOM Trio). Image courtesy of Dr. D. Parker, Advanced Research (Salt Lake City, UT).

will be detected that will otherwise be obscured by diffuse signal abnormalities. Furthermore, partial volume effects (e.g., between cortex and CSF) can be reduced, which improves the conspicuity of lesions as well as the accuracy of other techniques, such as fMRI. Conversely, the stronger signal at $3 \mathrm{~T}$ may be used to reduce scanning duration. Often a scan time reduction translates directly into higher image quality and is especially helpful when scanning uncooperative patients. In a clinical environment, this eventually leads to a higher patient throughput.

In addition to the promising advantage of greater SNR, higher fields also give rise to several challenges, which will be discussed in detail in the next paragraph. Most of these high-field effects depend on the application and may turn out to be advantages or disadvantages. For example, the stronger sensitivity to local susceptibilities in the tissue results in the well-known high sensitivity of fMRI at high field strength, making it one major reason to migrate to higher field strength. At the same time, however, susceptibility-related inhomogeneities at airtissue interfaces may cause severe degradation of the image quality in areas, such as the frontal or temporal lobe of the brain, and have to be addressed to compete with lower field image quality.

\section{Contrast (T1, T2, T2*-relaxation times)}

Compared with 1.5 Tesla, the $\mathrm{T} 1$ relaxation times for semi-solid tissues are significantly $(\sim 30 \%)$ longer at 3T. ${ }^{148-151}$ For example, T1 relaxation times in gray mat- 


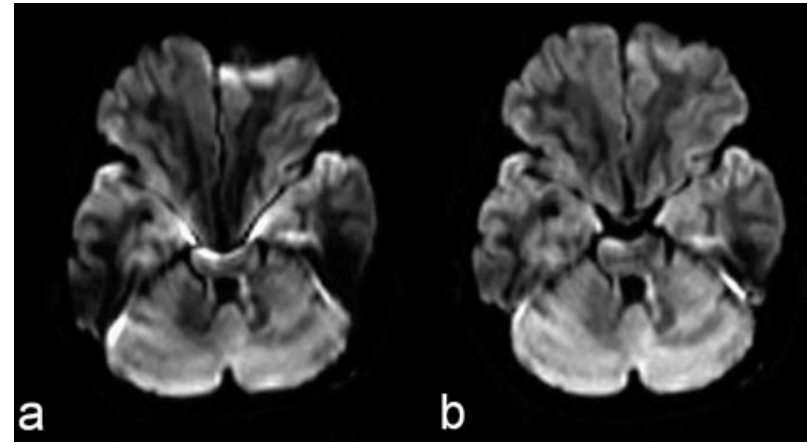

FIG. 27. Diffusion-weighted images $\left(b=1000 \mathrm{~s} / \mathrm{mm}^{2}\right)$ without acceleration (left) and with a parallel imaging acceleration factor 2 (right). Frontal and temporal brain tissue exhibit significantly reduced distortions and signal loss with the use of parallel imaging (single shot EPI, matrix $=128 \times 128, \mathrm{FOV}=220 \mathrm{~mm}, \mathrm{TE}=$ $80 \mathrm{~ms}$, 3T MAGNETOM Trio). Images courtesy of Prof. J. Frahm (MPI, Göttingen, Germany).

ter in the human brain are roughly $900 \mathrm{~ms}$ at $1.5 \mathrm{~T}$ and $1300 \mathrm{~ms}$ at 3T. For numerous imaging applications, the longer $\mathrm{T} 1$ necessitates protocol parameter changes, often resulting in longer total scan time, thereby reducing the potential gain in throughput. However, many MRI applications also benefit from the longer T1, such as MR angiography [e.g., time of flight (TOF)] techniques with and without contrast agents) and ASL techniques. The longer T1 leads to an improved suppression of background signals and provides a stronger signal enhancement from unsaturated spins moving into the imaging slice (FIG. 26).

In contrast to the longer $\mathrm{T} 1$ at higher fields, $\mathrm{T} 2$ stays roughly constant when comparing $1.5 \mathrm{~T}$ and $3.0 \mathrm{~T}$. Conversely, $\mathrm{T} 2 *$ is reduced at higher fields, which has particular impact on the choice of sequence parameters to reduce image artifacts and degradation of the pointspread function as a result of significant signal decay during the readout duration.

\section{Stronger susceptibility effects}

Local susceptibility gradients in the imaging volume cause phase dispersion of the spins and subsequently degradation of the received MRI signal. The respective phase-effects correlate with the magnetic field. Functional imaging by means of the BOLD contrast ${ }^{53}$ benefits from this stronger susceptibility effect as it provides improved contrast between functional states that can be characterized by differences in the local concentration of deoxyhemoglobin in the blood (FIGS. 24-25).

The same underlying mechanism, however, leads to unwanted degradation of the image quality in many applications. In particular, air-tissue interfaces often cause severe susceptibility artifacts resulting in stronger signal pile-up distortion or even a complete signal drop-out. In the brain, this is particularly present in the frontal lobe, posterior fossa, and auditory cortex. Susceptibility arti-

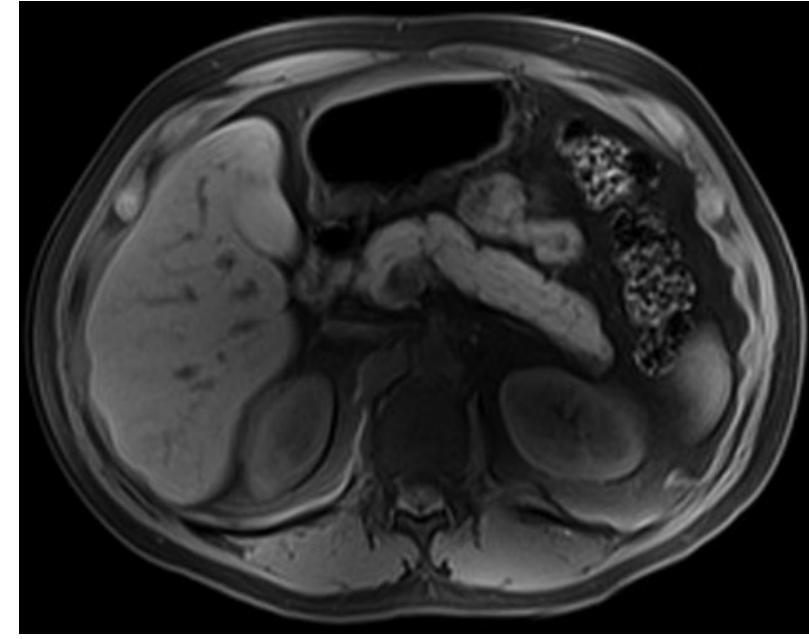

FIG. 28. T1-weighted FLASH $2 D$ image of the abdomen with fat saturation. $(\mathrm{TR}=132 \mathrm{~ms}, \mathrm{TE}=2.8 \mathrm{~ms}$, matrix $=154 \times 256$, FoV $=300 \times 400 \mathrm{~mm}^{2}$, acquisition time $=20 \mathrm{~s}$, 3T MAGNETOM Trio). Images courtesy of Silke Bosk (Siemens, Erlangen, Germany).

facts increase with the readout duration and voxel size (intra-voxel dephasing). Consequently, higher spatial resolution and methods that reduce the readout duration, such as multishot techniques, parallel imaging, and stronger gradients hardware systems can address this high-field challenge (FIG. 27).

\section{Improved spectral resolution}

The chemical shift $\delta \mathrm{B}_{0}$ in Hertz between different metabolites and the water-fat shift increases proportionally with the magnetic field. This, however, is impaired by the stronger sensitivity to susceptibility effects at higher fields, which leads to increased spectral line-width in MRS. Therefore, an excellent magnet homogeneity and improved shimming is mandatory for high-quality body imaging and spectroscopy at 3T (FIG. 28).

The improved spectral resolution at high fields also works in one's favor when doing spectral fat saturation. But again, the sensitivity to susceptibility effects at highfield works against this improvement. Therefore, it is not only for MRS but also for excellent fat saturation of great importance to have a good shimming system (FIG. 28). Moreover, fat suppression at high field sometimes does not work quite as efficiently due to B1 inhomogeneities. The desired chemical selective RF does not play out the desired flip angle and leaves behind a significant amount of unsaturated lipids that can spoil the image quality. To some extent, the sensitivity of chemical saturation pulses can be improved by using adiabatic RF pulses or by water-only excitation with spectral-spatial RF pulses. ${ }^{152}$

\section{Increased physiological noise}

The noise in MRI at clinical field strengths is typically dominated by the thermal noise. When scanning a sub- 


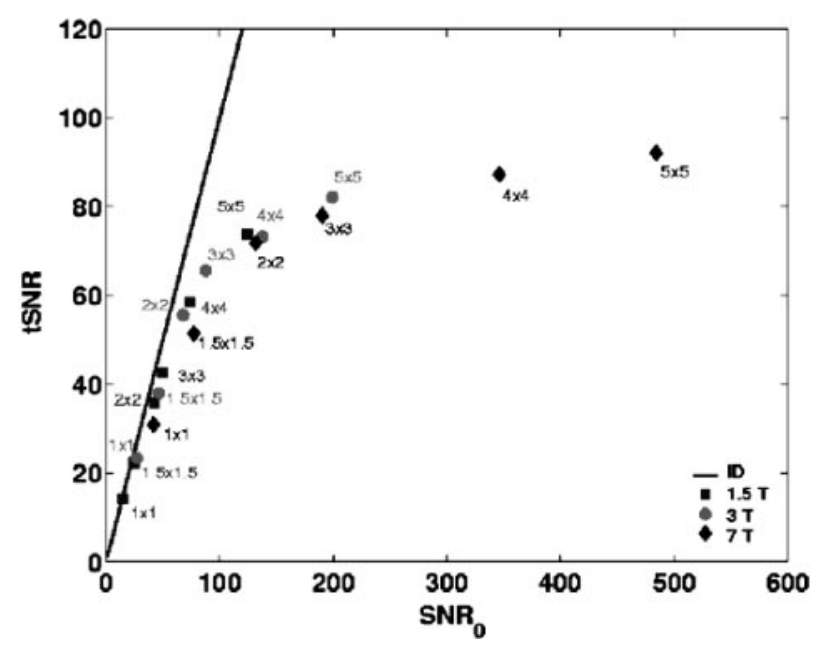

FIG. 29. Signal to Noise Ratio (SNR) in fMRI time series (tSNR) as a function of image SNR $\left(\mathrm{SNR}_{0}\right)$ for different spatial resolutions: this figure shows that the time series SNR at high-field strength is only exploited when going to smaller voxels. Changes in SNR were produced by varying the voxel volume. Labels indicate the in-plane resolution in $\mathrm{mm}^{2}$ at 3- $\mathrm{mm}$ slice thickness. Measurements were derived from areas of cortical gray matter and are averages over five subjects at each field strength. The solid line represents the line of identity $\left(\mathrm{tSNR}=\mathrm{SNR}_{0}\right)$. Data courtesy of C. Trianatfyllou (Martinos Center for Biomedical Imaging, Massachusetts General Hospital, Boston, MA).

ject, however, the so-called physiological noise increases the measurable image noise. ${ }^{153}$ In particular in BOLD, imaging strong physiological noise arises from respiration and cardiac cycles and changes in brain activity (blood flow, blood volume, and metabolism). In contrast to thermal noise, the physiological noise correlates with signal intensity and magnetic field strength. For fMRI at 3T, the physiological noise can easily dominate the total image noise, thereby reducing the expected gain in SNR with higher magnetic fields. Fortunately, a careful choice of sequence parameters can address this issue. For example, imaging at higher spatial resolution reduces the signal strength and minimizes the respective physiological noise components (FIG. 29).

\section{Increased RF power deposition}

The RF power needed to generate a certain B1 amplitude increases quadratically with the field strength. To keep tissue heating within safe levels, RF power is monitored carefully during the scanning procedure. The specific absorption rate (SAR, the energy deposited in the body per unit of mass) describes the distribution and amount of absorbed RF power per kilogram sample weight and is limited to $3.2 \mathrm{~W} / \mathrm{kg}$ and $4 \mathrm{~W} / \mathrm{kg}$ over a 6-min averaging period for head and body applications, respectively. ${ }^{154}$ Many RF-intense applications at 3T, such as sequences consisting of a frequent repetition of $180^{\circ}$ pulses (e.g., turbo spin echo sequences) may exceed the SAR limits. With current technology, however, the SAR limitation can be addressed by various techniques.

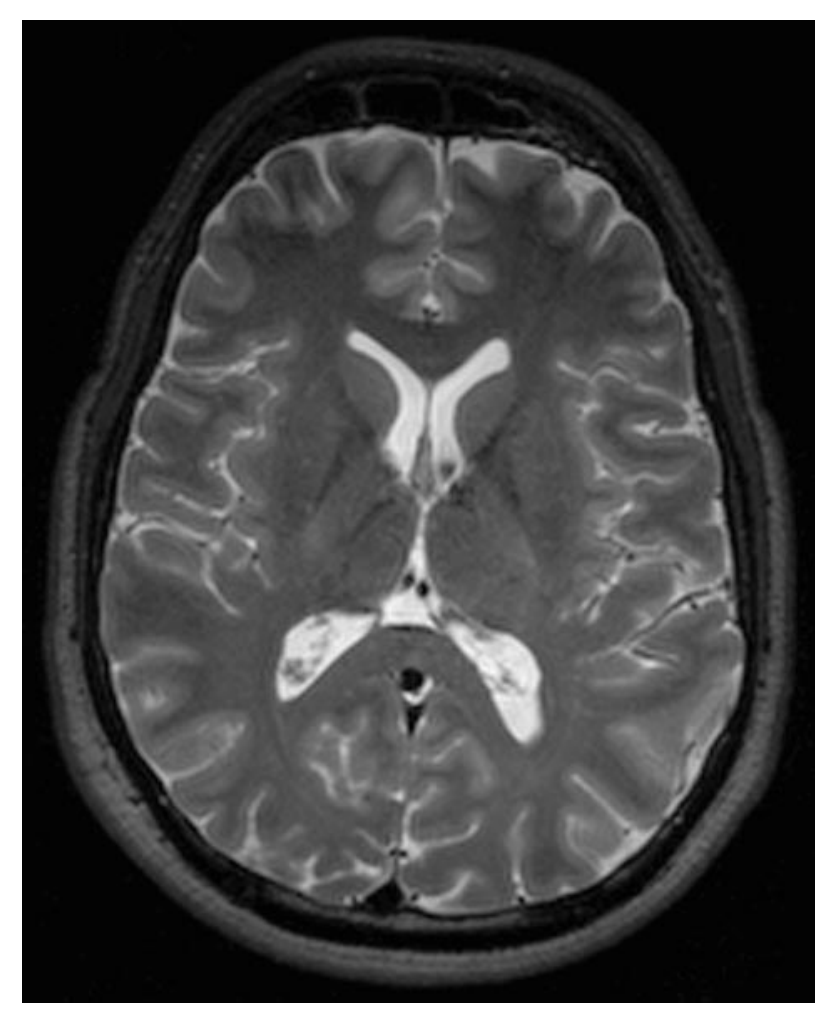

FIG. 30. 3D T2-weighted images acquired with the variable flip echo technique that allows an approximate SAR reduction of $25 \%$ (3D turbo spin echo sequence, matrix $=222 \times 256$, FOV $=$ $201 \times 230 \mathrm{~mm}$, TE $=366 \mathrm{~ms}$, acquisition time $=6: 45 \mathrm{~min}$, 3T MAGNETOM Trio). Images courtesy of Wilhelm Horger (Siemens, Erlangen, Germany).

When SNR is not the limiting factor, parallel imaging can be used in many applications to reduce the RF power by a factor of two or more. Specific RF pulse designs may also help to overcome this problem. Specifically, the hyperecho ${ }^{155}$ and variable flip angle techniques ${ }^{156}$ allow for significant SAR reduction without compromising imaging time and quality (FIG. 30).

\section{Reduced B1 uniformity}

At high magnetic fields, the RF wavelength is reduced and, within tissue, becomes close to the physical dimensions of the human body. This causes a reduced B1 uniformity, i.e., a spatial distribution of flip angles at 3T. In more detail, both sample properties (dielectric constant and electric conductivity) and dimensions determine local B1 focusing (signal pile) or B1 shielding (signal loss) effects that might appear as brightness or contrast variations. Furthermore, the signal reception may be affected by an enhanced distribution of the spatial sensitivity leading to additional brightness variations. Therefore, the received MRI signal at high fields exhibits a stronger dependency on the spatial sensitivity and flip angle distribution than similar scans at 1.5T. Recently, it has been shown that the contrast between gray and white matter increases with rising magnetic field strength and 
that for particular pulse sequences, such as spoiled gradient echo sequences in combination with transmit/receive coils and larger flip angles, the B1 heterogeneity is negligible. ${ }^{157}$

Overall, the spatial variation in signal intensity is rather complex nature and difficult to control. However, certain techniques have been proposed to reduce B1 nonuniformity, such as the use of optimized sequences, dielectric pillows, ${ }^{158}$ and parallel transmit techniques. ${ }^{159}$

\section{Acoustic noise}

Acoustic noise also represents a topic to be considered when moving to higher magnetic fields. The acoustic noise is closely related to the force on the gradient coil that arises when field gradients are switched for signal encoding. This force increases with the magnetic field. This can be a serious inconvenience for the patient and a problem for certain applications (e.g., pediatric imaging) at high magnetic fields.

For patient safety, acoustic noise produced by the MRI scanner is limited to less than $100 \mathrm{~dB},{ }^{154}$ which is usually satisfied with ear protection. Respecting these safety concerns has typically been achieved by insulating materials. In addition, future hardware modifications and applications, such as parallel imaging, are capable and already greatly reduce this issue. To some extent, gradient waveforms can be designed in a way that reduces the $\mathrm{dB} / \mathrm{dt}$ of both the attack and decay ramps and, hence, minimizing the acoustic burden.

\section{Summary}

Over the past few years, MRI at 3T has clearly evolved into a clinical tool. Initially, image quality in various applications had been compromised by many challenges, such as SAR, susceptibility artifacts, and B1 nonuniformity. It is fascinating, however, to see that these challenges were similar to the challenges faced at $1.5 \mathrm{~T}$ field strength at the end of the 1980s. A strong focus on new developments and optimization of high field applications from vendors and researchers has addressed many of these challenges. A particularly important tool in this scenario is parallel imaging, as it allows one to address image artifacts from susceptibility effects, reduce acoustic noise of the scanner, and greatly reduce SAR. Other techniques, such as specially designed RF-pulses, hyperecho and variable flip angle (VFL) techniques are also a vital complement to reduce the SAR even further. As of today, head and musculoskeletal applications at 3T clearly demonstrate the gain in spatial or temporal resolution from higher fields. However, some body and cardiac applications are still challenged by the aforementioned problems found at higher magnetic field strengths. Although feasible, their robustness in particular against B1 uniformity and susceptibility effects needs improve- ment. Further technical developments, such as parallel imaging and parallel transmit technique, represent promising approaches. Today, more than two-thirds of the field strength chosen world-wide is 1.5T. Extrapolating from the achieved improvements in MRI at higher fields, $3 \mathrm{~T}$ has a bright future ahead with these new avenues.

\section{CONCLUSIONS}

Advanced MRI techniques continue to show promising results for the diagnostic evaluation of patients with various diseases and will facilitate treatment decisions and therapeutic monitoring. Most of the methods presented in this manuscript can now be applied on most state-of-the-art MRI scanners, although some cuttingedge material is still under development and additional time might be required until this technology is commonly available.

The MRI techniques presented here have led to a tremendous increase in the range of information that can be obtained during an MRI session in addition to conventional structural MRI. It is foreseeable that many more technical improvements will occur that may further help improve diagnosis and treatment.

Acknowledgments: This work was supported in part by the National Institute of Health (NIH-1R01EB2711), Fonds voor Wetenschappelijk Onderzoek Vlaanderen, the Oak Foundation, the Center of Advanced MR Technology of Stanford (NCRR P41 RR 09784), and the Lucas Foundation.

\section{REFERENCES}

1. Crank J. The mathematics of diffusion. New York: Oxford University Press, 1956.

2. Basser PJ, Mattiello J, LeBihan D. Estimation of the effective self-diffusion tensor from the NMR spin echo. J Magn Reson 103:247-254, 1994

3. Basser PJ. Inferring microstructural features and the physiological state of tissues from diffusion-weighted images. NMR Biomed 8:333-344, 1995.

4. Moseley ME, Kucharczyk J, Mintorovitch J, Cohen Y, Kurhanewicz J, Derugin N, et al. Diffusion-weighted MR imaging of acute stroke: Correlation with T2-weighted and magnetic susceptibility-enhanced MR imaging in cats. AJNR Am J Neuroradiol 11:423-429, 1990.

5. Lansberg MG, Norbash AM, Marks MP, Tong DC, Moseley ME, Albers GW. Advantages of adding diffusion-weighted magnetic resonance imaging to conventional magnetic resonance imaging for evaluating acute stroke. Arch Neurol 57:1311-1316, 2000.

6. Sá de Camargo EC, Koroshetz WJ. Neuroimaging of ischemia and infarction. NeuroRx 2:265-276, 2005.

7. Bammer R, Fazekas F. Diffusion imaging in multiple sclerosis. Neuroimaging Clin N Am 12:71-106, 2002.

8. Bakshi R, Minagar A, Jaisani Z, Wolinsky JS. Imaging of multiple sclerosis: role in neurotherapeutics. NeuroRx 2:277-303, 2005.

9. Toosy AT, Werring DJ, Orrell RW, Howard RS, King MD, Barker GJ, et al. Diffusion tensor imaging detects corticospinal tract involvement at multiple levels in amyotrophic lateral sclerosis. J Neurol Neurosurg Psychiatry 74:1250-1257, 2003.

10. Bammer R, Acar B, Moseley ME. In vivo MR tractography using diffusion imaging. Eur J Radiol 45:223-234, 2003. 
11. Pierpaoli C, Jezzard P, Basser PJ, Barnett A, Di Chiro G. Diffusion tensor MR imaging of the human brain. Radiology 201:637648, 1996.

12. Merboldt KD, Hanicke W, Bruhn H, Gyngell ML, Frahm J. Diffusion imaging of the human brain in vivo using high-speed steam MRI. Magn Reson Med 23:179-192, 1992.

13. Buxton RB. The diffusion sensitivity of fast steady-state free precession imaging. Magn Reson Med 29:235-243, 1993.

14. Le Bihan D, Turner R, MacFall JR. Effects of intravoxel incoherent motions (IVIM) in steady-state free precession (SSFP) imaging: application to molecular diffusion imaging. Magn Reson Med 10:324-337, 1989.

15. Gudbjartsson H, Patz S. Simultaneous calculation of flow and diffusion sensitivity in steady-state free precession imaging. Magn Reson Med 34:567-579, 1995.

16. Bammer R. Basic principles of diffusion-weighted imaging. Eur J Radiol 45:169-184, 2003.

17. Skare S, Andersson JL. On the effects of gating in diffusion imaging of the brain using single shot EPI. Magn Reson Imaging 19:1125-1128, 2001.

18. Poncelet BP, Wedeen VJ, Weisskoff RM, Cohen MS. Brain parenchyma motion: measurement with cine echo-planar MR imaging. Radiology 185:645-651, 1992.

19. Anderson AW, Gore JC. Analysis and correction of motion artifacts in diffusion weighted imaging. Magn Reson Med 32:379387, 1994

20. Butts K, de Crespigny A, Pauly JM, Moseley M. Diffusionweighted interleaved echo-planar imaging with a pair of orthogonal navigator echoes. Magn Reson Med 35:763-770, 1996.

21. Bammer R, Stollberger R, Augustin M, Simbrunner J, Offenbacher H, Kooijman H, et al. Diffusion-weighted imaging with navigated interleaved echo-planar imaging and a conventional gradient system. Radiology 211:799-806, 1999.

22. Butts K, Pauly J, de Crespigny A, Moseley M. Isotropic diffusion-weighted and spiral-navigated interleaved EPI for routine imaging of acute stroke. Magn Reson Med 38:741-749, 1997.

23. Atkinson D, Porter DA, Hill DL, Calamante F, Connelly A. Sampling and reconstruction effects due to motion in diffusionweighted interleaved echo planar imaging. Magn Reson Med 44:101-109, 2000.

24. de Crespigny AJ, Marks MP, Enzmann DR, Moseley ME. Navigated diffusion imaging of normal and ischemic human brain. Magn Reson Med 33:720-728, 1995.

25. Jackson JI, Meyer CH, Nishimura DH, Macovski A. Selection of a convolution function for fourier inversion using gridding. IEEE Trans Med Imag 10:473-478, 1991.

26. Miller KL, Pauly JM. Nonlinear phase correction for navigated diffusion imaging. Magn Reson Med 50:343-353, 2003.

27. Mansfield P. Multi-planar image formation using NMR spin echoes. J Phys C 10:L55-L58, 1977.

28. Farzaneh F, Riederer SJ, Pelc NJ. Analysis of T2 limitations and off-resonance effects on spatial resolution and artifacts in echoplanar imaging. Magn Reson Med 14:123-139, 1990.

29. Cercignani M, Horsfield MA, Agosta F, Filippi M. Sensitivityencoded diffusion tensor MR imaging of the cervical cord. AJNR Am J Neuroradiol 24:1254-1256, 2003.

30. Bammer R, Keeling SL, Augustin M, Pruessmann KP, Wolf R, Stollberger R, et al. Improved diffusion-weighted single-shot echo-planar imaging (EPI) in stroke using sensitivity encoding (SENSE). Magn Reson Med 46:548-554, 2001.

31. Bammer R, Auer M, Keeling SL, Augustin M, Stables LA, Prokesch RW, et al. Diffusion tensor imaging using single-shot SENSE-EPI. Magn Reson Med 48:128-136, 2002.

32. Jezzard P, Balaban RS. Correction for geometric distortion in echo planar images from B0 field variations. Magn Reson Med 34:65-73, 1995.

33. Andersson JL, Skare S. A model-based method for retrospective correction of geometric distortions in diffusion-weighted EPI. Neuroimage 16:177-199, 2002.

34. Andersson JL, Skare S, Ashburner J. How to correct susceptibility distortions in spin-echo echo-planar images: application to diffusion tensor imaging. Neuroimage 20:870-888, 2003.
35. Jezzard P, Barnett AS, Pierpaoli C. Characterization of and correction for eddy current artifacts in echo planar diffusion imaging. Magn Reson Med 39:801-812, 1998.

36. Reese TG, Heid O, Weisskoff RM, Wedeen VJ. Reduction of eddy-current-induced distortion in diffusion MRI using a twicerefocused spin echo. Magn Reson Med 49:177-182, 2003.

37. Bammer R, Auer M. Correction of eddy-current induced image warping in diffusion-weighted single-shot EPI using constrained non-rigid mutual information image registration. Paper presented at the 9th Annual Meeting of the International Society of Magnetic Resonance in Medicine, Glasgow, Scotland, p 508, 2001.

38. de Crespigny A, Moseley ME. Eddy current induced image warping induced in diffusion weighted EPI. Paper presented at the 6th Annual Meeting of the International Society of Magnetic Resonance in Medicine, Sydney, Australia, p 661, 1998.

39. Haselgrove JC, Moore JR. Correction for distortion of echoplanar images used to calculate the apparent diffusion coefficient. Magn Reson Med 36:960-964, 1996.

40. Glover GH, Pauly JM. Projection reconstruction techniques for reduction of motion effects in MRI. Magn Reson Med 28:275289, 1992.

41. Gmitro AF, Alexander AL. Use of a projection reconstruction method to decrease motion sensitivity in diffusion-weighted MRI. Magn Reson Med 29:835-838, 1993.

42. Trouard TP, Sabharwal Y, Altbach MI, Gmitro AF. Analysis and comparison of motion-correction techniques in diffusionweighted imaging. J Magn Reson Imaging 6:925-935, 1996.

43. Pipe JG, Farthing VG, Forbes KP. Multishot diffusion-weighted FSE using propeller MRI. Magn Reson Med 47:42-52, 2002.

44. Forbes KP, Pipe JG, Karis JP, Heiserman JE. Improved image quality and detection of acute cerebral infarction with propeller diffusion-weighted MR imaging. Radiology 225:551-555, 2002.

45. Li TQ, Takahashi AM, Hindmarsh T, Moseley ME. ADC mapping by means of a single-shot spiral MRI technique with application in acute cerebral ischemia. Magn Reson Med 41:143-147, 1999.

46. Bammer R, Glover GH, Moseley ME. Diffusion tensor spiral imaging. Paper presented at the 10th Annual Meeting of the International Society of Magnetic Resonance in Medicine, Honolulu, HI, p 1111, 2002.

47. Glover GH. Basic and advanced concepts of spiral imaging. Paper presented at the International Society for Magnetic Resonance in Medicine Fast MRI Workshop, Asilomar, CA, pp 115-119, 1997.

48. Glover GH, Lai S. Self-navigated spiral fMRI: interleaved versus single-shot. Magn Reson Med 39:361-368, 1998.

49. Spielman DM, Pauly JM, Meyer CH. Magnetic resonance fluoroscopy using spirals with variable sampling densities. Magn Reson Med 34:388-394, 1995.

50. Kim DH, Adalsteinsson E, Spielman DM. Simple analytic variable density spiral design. Magn Reson Med 50:214-219, 2003.

51. Liu C, Bammer R, Kim DH, Moseley ME. Self-navigated interleaved spiral (SNAILS): application to high-resolution diffusion tensor imaging. Magn Reson Imaging 52:1388-1396, 2004.

52. Deleted in proof.

53. Ogawa S, Lee TM, Nayak AS, Glynn P. Oxygenation-sensitive contrast in magnetic resonance image of rodent brain at high magnetic fields. Magn Reson Med 14:68-78, 1990.

54. Dejerine JJ. Anatomie des centres nerveux. Paris: Rueff, 1895.

55. Krieg WJS. Connections of the cerebral cortex. Evanston, IL: Brain Books, 1963.

56. Krieg WJS. Architectonics of human cerebral fiber systems. Evanston, IL: Brain Books, 1973.

57. Pribam K, MacLean P. Neuronographic analysis of medial and basal cerebral cortex. J Neurophysiol 16:324-340, 1953.

58. Whitlock DG, Nauta WJH. Subcortical projections from temporal neocortex in macaca mulatto. J Comp Neurol 106:183-212, 1956.

59. Turner BH, Mishkin M, Knapp M. Organization of the amygdalopetal projections from modality-specific cortical association areas in the monkey. J Comp Neurol 191:515-543, 1980.

60. Yagishita A, Nakano I, Oda M, Hirano A. Location of the corticospinal tract in the internal capsule at MR imaging. Radiology 191:455-460, 1994. 
61. Godement P, Vanselow J, Thanos S, Bonhoeffer F. A study in developing visual systems with a new method of staining neurones and their processes in fixed tissue. Development 101:697713, 1987

62. Mori S, Crain BJ, Chacko VP, van Zijl PC. Three-dimensional tracking of axonal projections in the brain by magnetic resonance imaging. Ann Neurol 45:265-269, 1999.

63. Conturo TE, Lori NF, Cull TS, Akbudak E, Snyder AZ, Shimony JS, et al. Tracking neuronal fiber pathways in the living human brain. Proc Natl Acad Sci 96:10422-10427, 1999.

64. Basser PJ, Pajevic S, Pierpaoli C, Duda J, Aldroubi A. In vivo fiber tractography using DT-MRI data. Magn Reson Med 44:625632, 2000.

65. Tench CR, Morgan PS, Wilson M, Blumhardt LD. White matter mapping using diffusion tensor MRI. Magn Reson Med 47:967972, 2002.

66. Tench CR, Morgan PS, Blumhardt LD, Constantinescu C. Improved white matter fiber tracking using stochastic labeling. Magn Reson Med 48:677-683, 2002.

67. Koch MA, Norris DG, Hund-Georgiadis M. An investigation of functional and anatomical connectivity using magnetic resonance imaging. Neuroimage 16:241-250, 2002.

68. Hagmann P, Thiran JP, Jonasson L, Vandergheynst P, Clarke S, Maeder P, et al. DTI mapping of human brain connectivity: statistical fibre tracking and virtual dissection. Neuroimage 19: 545-554, 2003.

69. Baird AE, Warach S. Magnetic resonance imaging of acute stroke. J Cereb Blood Flow Metab 18:583-609, 1998.

70. Calamante F, Thomas DL, Pell GS, Wiersma J, Turner R. Measuring cerebral blood flow using magnetic resonance imaging techniques. J Cereb Blood Flow Metab 19:701-735, 1999.

71. Brooks DJ. Positron emission tomography and single-photon emission computed tomography in central nervous system drug development. NeuroRx 2:226-236, 2005.

72. Villringer A, Rosen BR, Belliveau JW, Ackerman JL, Lauffer $\mathrm{RB}$, Buxton RB, et al. Dynamic imaging with lanthanide chelates in normal brain: Contrast due to magnetic susceptibility effects. Magn Reson Med 6:164-174, 1988.

73. Fisel CR, Ackerman JL, Buxton RB, Garrido L, Belliveau JW, Rosen BR, et al. MR contrast due to microscopically heterogeneous magnetic susceptibility: Numerical simulations and applications to cerebral physiology. Magn Reson Med 17:336-347, 1991.

74. Belliveau JW, Rosen BR, Kantor HL, Rzedzian RR, Kennedy DN, McKinstry RC, et al. Functional cerebral imaging by susceptibility-contrast NMR. Magn Reson Med 14:538-546, 1990.

75. Reichenbach JR, Hacklander T, Harth T, Hofer M, Rassek M, Modder U. ${ }^{1} \mathrm{H} \mathrm{T} 1$ and T2 measurements of the MR imaging contrast agents GD-DTPA and GD-DTPA BMA at 1.5T. Eur Radiol 7:264-274, 1997.

76. Knight RA, Ordidge RJ, Helpern JA, Chopp M, Rodolosi LC, Peck D. Temporal evolution of ischemic damage in rat brain measured by proton nuclear magnetic resonance imaging. Stroke 22:802-808, 1991.

77. Helpern JA, Dereski MO, Knight RA, Ordidge RJ, Chopp M, Qing ZX. Histopathological correlations of nuclear magnetic resonance imaging parameters in experimental cerebral ischemia. Magn Reson Imaging 11:241-246, 1993.

78. Meier P, Zierler KL. On the theory of the indicator-dilution method for measurement of blood flow and volume. J Appl Physiol 6:731-744, 1954.

79. Weisskoff RM, Chesler D, Boxerman JL, Rosen BR. Pitfalls in MR measurement of tissue blood flow with intravascular tracers: which mean transit time? Magn Reson Med 29:553-558, 1993.

80. Lassen NA. Cerebral transit of an intravascular tracer may allow measurement of regional blood volume but not regional blood flow. J Cereb Blood Flow Metab 4:633-634, 1984.

81. Grandin CB, Duprez TP, Smith AM, Oppenheim C, Peeters A, Robert AR, et al. Which MR-derived perfusion parameters are the best predictors of infarct growth in hyperacute stroke? Comparative study between relative and quantitative measurements. $R a$ diology 223:361-370, 2002.

82. Ostergaard L, Sorensen AG, Kwong KK, Weisskoff RM, Gyl- densted C, Rosen BR. High resolution measurement of cerebral blood flow using intravascular tracer bolus passages. Part II: experimental comparison and preliminary results. Magn Reson Med 36:726-736, 1996.

83. Ostergaard L, Weisskoff RM, Chesler DA, Gyldensted C, Rosen BR. High resolution measurement of cerebral blood flow using intravascular tracer bolus passages. Part I: mathematical approach and statistical analysis. Magn Reson Med 36:715-725, 1996.

84. Ostergaard L, Johannsen P, Host-Poulsen P, Vestergaard-Poulsen $\mathrm{P}$, Asboe H, Gee AD, et al. Cerebral blood flow measurements by magnetic resonance imaging bolus tracking: comparison with [(15)O]H2O positron emission tomography in humans. J Cereb Blood Flow Metab 18:935-940, 1998.

85. Ostergaard L, Smith DF, Vestergaard-Poulsen P, Hansen SB, Gee AD, Gjedde A, et al. Absolute cerebral blood flow and blood volume measured by magnetic resonance imaging bolus tracking: comparison with positron emission tomography values. J Cereb Blood Flow Metab 18:425-432, 1998.

86. Lin W, Celik A, Derdeyn C, An H, Lee Y, Videen T, et al. Quantitative measurements of cerebral blood flow in patients with unilateral carotid artery occlusion: a PET and MR study. J Magn Reson Imaging 14:659-667, 2001.

87. Calamante F, Gadian DG, Connelly A. Delay and dispersion effects in dynamic susceptibility contrast MRI: simulations using singular value decomposition. Magn Reson Med 44:466-473, 2000.

88. Detre JA, Leigh JS, Williams DS, Koretsky AP. Perfusion imaging. Magn Reson Med 23:37-45, 1992.

89. Detre JA, Zhang W, Roberts DA, Silva AC, Williams DS, Grandis DJ, et al. Tissue specific perfusion imaging using arterial spin labeling. NMR Biomed 7:75-82, 1994.

90. Williams DS, Detre JA, Leigh JS, Koretsky AP. Magnetic resonance imaging of perfusion using spin inversion of arterial water. Proc Natl Acad Sci 89:212-216, 1992.

91. Alsop DC, Detre JA. Multisection cerebral blood flow MR imaging with continuous arterial spin labeling. Radiology 208:410416, 1998.

92. Edelman RR, Siewert B, Darby DG, Thangaraj V, Nobre AC, Mesulam MM, et al. Qualitative mapping of cerebral blood flow and functional localization with echo-planar MR imaging and signal targeting with alternating radio frequency. Radiology 192: 513-520, 1994.

93. Pruessmann KP, Golay X, Stuber M, Scheidegger MB, Boesiger P. RF pulse concatenation for spatially selective inversion. $J$ Magn Reson 146:58-65, 2000.

94. Golay X, Stuber M, Pruessmann KP, Meier D, Boesiger P. Transfer insensitive labeling technique (TILT): application to multislice functional perfusion imaging. J Magn Reson Imaging 9:454-461, 1999.

95. Wong E, Buxton R, Frank L. Quantitative perfusion imaging using epistar and fair. Paper presented at the 4th Annual Meeting of the International Society for Magnetic Resonance in Medicine, New York, NY, p 8, 1996.

96. Jahng GH, Zhu XP, Matson GB, Weiner MW, Schuff N. Improved perfusion-weighted MRI by a novel double inversion with proximal labeling of both tagged and control acquisitions. Magn Reson Med 49:307-314, 2003.

97. Kim SG. Quantification of relative cerebral blood flow change by flow-sensitive alternating inversion recovery (FAIR) technique: application to functional mapping. Magn Reson Med 34:293-301, 1995.

98. Helpern JA, Branch CA, Yongbi MN, Huang NC. Perfusion imaging by un-inverted flow-sensitive alternating inversion recovery (UNFAIR). Magn Reson Imaging 15:135-139, 1997.

99. Mai VM, Hagspiel KD, Christopher JM, Do HM, Altes T, Knight-Scott J, et al. Perfusion imaging of the human lung using flow-sensitive alternating inversion recovery with an extra radiofrequency pulse (FAIRER). Magn Reson Imaging 17:355-361, 1999.

100. Lai S, Wang J, Jahng GH. Fair exempting separate T (1) measurement (FAIREST): a novel technique for online quantitative perfusion imaging and multi-contrast fMRI. NMR Biomed 14: 507-516, 2001. 
101. Wong EC, Buxton RB, Frank LR. Implementation of quantitative perfusion imaging techniques for functional brain mapping using pulsed arterial spin labeling. NMR Biomed 10:237-249, 1997.

102. Detre JA, Wang J. Technical aspects and utility of fMRI using BOLD and ASL. Clin Neurophysiol 113:621-634, 2002.

103. Calamante F, Gadian DG, Connelly A. Quantification of perfusion using bolus tracking magnetic resonance imaging in stroke: Assumptions, limitations, and potential implications for clinical use. Stroke 33:1146-1151, 2002.

104. Sorensen AG, Buonanno FS, Gonzalez RG, Schwamm LH, Lev MH, Huang-Hellinger FR, et al. Hyperacute stroke: evaluation with combined multisection diffusion-weighted and hemodynamically weighted echo-planar MR imaging. Radiology 199:391401, 1996

105. Beaulieu C, de Crespigny A, Tong DC, Moseley ME, Albers GW, Marks MP. Longitudinal magnetic resonance imaging study of perfusion and diffusion in stroke: evolution of lesion volume and correlation with clinical outcome. Ann Neurol 46:568-578, 1999.

106. Barber PA, Darby DG, Desmond PM, Yang Q, Gerraty RP, Jolley $\mathrm{D}$, et al. Prediction of stroke outcome with echoplanar perfusionand diffusion- weighted mri. Neurology 51:418-426, 1998.

107. Schlaug G, Benfield A, Baird AE, Siewert B, Lovblad KO, Parker $\mathrm{RA}$, et al. The ischemic penumbra: operationally defined by diffusion and perfusion MRI. Neurology 53:1528-1537, 1999.

108. Tong DC, Yenari MA, Albers GW, O'Brien M, Marks MP, Moseley ME. Correlation of perfusion- and diffusion-weighted MRI with NIHSS score in acute (6.5 hour) ischemic stroke. Neurology 50:864-870, 1998.

109. Neumann-Haefelin T, Wittsack HJ, Wenserski F, Siebler M, Seitz RJ, Modder U, et al. Diffusion- and perfusion-weighted MRI. The DWI/PWI mismatch region in acute stroke. Stroke 30:1591-1597, 1999.

110. Baird AE, Lovblad KO, Dashe JF, Connor A, Burzynski C, Schlaug G, et al. Clinical correlations of diffusion and perfusion lesion volumes in acute ischemic stroke. Cerebrovasc Dis 10: 441-448, 2000.

111. Schwamm LH, Koroshetz WJ, Sorensen AG, Wang B, Copen WA, Budzik R, et al. Time course of lesion development in patients with acute stroke: Serial diffusion- and hemodynamicweighted magnetic resonance imaging. Stroke 29:2268-2276, 1998.

112. Baird AE, Benfield A, Schlaug G, Siewert B, Lovblad KO, Edelman RR, et al. Enlargement of human cerebral ischemic lesion volumes measured by diffusion-weighted magnetic resonance imaging. Ann Neurol 41:581-589, 1997.

113. Karonen JO, Liu Y, Vanninen RL, Ostergaard L, Kaarina Partanen PL, Vainio PA, et al. Combined perfusion- and diffusionweighted MR imaging in acute ischemic stroke during the 1st week: a longitudinal study. Radiology 217:886-894, 2000.

114. Warach S, Pettigrew LC, Dashe JF, Pullicino P, Lefkowitz DM, Sabounjian L, et al. Effect of citicoline on ischemic lesions as measured by diffusion- weighted magnetic resonance imaging. Citicoline 010 investigators. Ann Neurol 48:713-722, 2000.

115. Sorensen AG, Copen WA, Ostergaard L, Buonanno FS, Gonzalez RG, Rordorf G, et al. Hyperacute stroke: simultaneous measurement of relative cerebral blood volume, relative cerebral blood flow, and mean tissue transit time. Radiology 210:519-527, 1999.

116. Darby DG, Barber PA, Gerraty RP, Desmond PM, Yang Q, Parsons M, et al. Pathophysiological topography of acute ischemia by combined diffusion- weighted and perfusion MRI. Stroke 30:2043-2052, 1999.

117. Chalela JA, Kang DW, Luby M, Ezzeddine M, Latour LL, Todd $\mathrm{JW}$, et al. Early magnetic resonance imaging findings in patients receiving tissue plasminogen activator predict outcome: insights into the pathophysiology of acute stroke in the thrombolysis era. Ann Neurol 55:105-112, 2004.

118. Parsons MW, Barber PA, Darby DG, Tress BM, Donnan GA, Davis SM. Hyperacute stroke diffusion- and perfusion-weighted MRI distinguishes t-Pa responders. Stroke 32(Suppl) 324:P181, 2001.

119. Kelly PJ, Hedley-Whyte ET, Primavera J, He J, Gonzalez RG. Diffusion MRI in ischemic stroke compared to pathologically verified infarction. Neurology 56:914-920, 2001.
120. Karonen JO, Nuutinen J, Kuikka JT, Vanninen EJ, Vanninen RL, Partanen PL, et al. Combined SPECT and diffusion-weighted MRI as a predictor of infarct growth in acute ischemic stroke. J Nucl Med 41:788-794, 2000.

121. Neumann-Haefelin T, Wittsack HJ, Fink GR, Wenserski F, Li TQ, Seitz RJ, et al. Diffusion- and perfusion-weighted MRI: influence of severe carotid artery stenosis on the DWI/PWI mismatch in acute stroke. Stroke 31:1311-1317, 2000.

122. Kohno K, Hoehn-Berlage M, Mies G, Back T, Hossmann KA. Relationship between diffusion-weighted MR images, cerebral blood flow, and energy state in experimental brain infarction. Magn Reson Imaging 13:73-80, 1995.

123. Mintorovitch J, Moseley ME, Chileuitt L, Shimizu H, Cohen Y, Weinstein PR. Comparison of diffusion- and T2-weighted MRI for the early detection of cerebral ischemia and reperfusion in rats. Magn Reson Med 18:39-50, 1991.

124. Rother J, de Crespigny AJ, D'Arceuil H, Iwai K, Moseley ME. Recovery of apparent diffusion coefficient after ischemia-induced spreading depression relates to cerebral perfusion gradient. Stroke 27:980-986, 1996.

125. Busch E, Kruger K, Allegrini PR, Kerskens CM, Gyngell ML, Hoehn-Berlage M, et al. Reperfusion after thrombolytic therapy of embolic stroke in the rat: Magnetic resonance and biochemical imaging. J Cereb Blood Flow Metab 18:407-418, 1998.

126. Li F, Han SS, Tatlisumak T, Liu KF, Garcia JH, Sotak CH, et al. Reversal of acute apparent diffusion coefficient abnormalities and delayed neuronal death following transient focal cerebral ischemia in rats. Ann Neurol 46:333-342, 1999.

127. Li F, Silva MD, Sotak CH, Fisher M. Temporal evolution of ischemic injury evaluated with diffusion-, perfusion-, and T2weighted MRI. Neurology 54:689-696, 2000.

128. Kidwell CS, Saver JL, Mattiello J, Starkman S, Vinuela F, Duckwiler G, et al. Thrombolytic reversal of acute human cerebral ischemic injury shown by diffusion/perfusion magnetic resonance imaging. Ann Neurol 47:462-469, 2000.

129. Marks MP, de Crespigny A, Lentz D, Enzmann DR, Albers GW, Moseley ME. Acute and chronic stroke: navigated spin-echo diffusion-weighted MR imaging. Radiology 199:403-408, 1996.

130. Neumann-Haefelin T, Wittsack HJ, Wenserski F, Li TQ, Moseley ME, Siebler M, et al. Diffusion- and perfusion-weighted MRI in a patient with a prolonged reversible ischaemic neurological deficit. Neuroradiology 42:444-447, 2000.

131. Lecouvet FE, Duprez TP, Raymackers JM, Peeters A, Cosnard G. Resolution of early diffusion-weighted and flair MRI abnormalities in a patient with TIA. Neurology 52:1085-1087, 1999.

132. Krueger K, Kugel H, Grond M, Thiel A, Maintz D, Lackner K. Late resolution of diffusion-weighted MRI changes in a patient with prolonged reversible ischemic neurological deficit after thrombolytic therapy. Stroke 31:2715-2718, 2000.

133. Uno M, Harada M, Okada T, Nagahiro S. Diffusion-weighted and perfusion-weighted magnetic resonance imaging to monitor acute intra-arterial thrombolysis. J Stroke Cerebrovascular Diseases 9:113-120, 2000.

134. Kidwell CS, Alger JR, Di Salle F, Starkman S, Villablanca P, Bentson J, Saver JL. Diffusion MRI in patients with transient ischemic attacks. Stroke 30:1174-1180, 1999.

135. Kidwell CS, Saver JL, Mattiello J, Alger JR, Starkman S, Duckwiler $\mathrm{G}$, et a. A diffusion-perfusion MRI signature predicting hemorrhagic transformation following intra-arterial thrombolysis. Stroke 32:S318, 2001.

136. Kidwell CS, Saver JL, Starkman S, Duckwiler G, Jahan R, Vespa $\mathrm{P}$, Villablanca JP, et al. Late secondary ischemic injury in patients receiving intraarterial thrombolysis. Ann Neurol 52:698-703, 2002.

137. Tong DC, Adami A, Moseley ME, Marks MP. Relationship between apparent diffusion coefficient and subsequent hemorrhagic transformation following acute ischemic stroke. Stroke 31:2378 2384, 2000.

138. Jacobs AH, Kracht LW, Gossmann A, Rüger MA, Thomas AV, Thiel A, Herholz K. Imaging in neurooncology. NeuroRx 2:333347, 2005. 
139. Dickerson BC, Sperling RA. Neuroimaging biomarkers for clinical trials of disease-modifying therapies in Alzheimer's disease. NeuroRx 2:348-360, 2005 .

140. Thulborn KR, Waterton JC, Matthews PM, Radda GK. Oxygenation dependence of the transverse relaxation time of water protons in whole blood at high field. Biochim Biophys Acta 714:265$270,1982$.

141. Turner R, Le Bihan D, Moonen CT, Despres D, Frank J. Echoplanar time course MRI of cat brain oxygenation changes. Magn Reson Med 22:159-166, 1991.

142. Kwong K, Belliveau J, Chesler D, Goldberg I. Dynamic magnetic resonance imaging of human brain activity during primary sensory stimulation. Proc Natl Acad Sci USA 89:5675-5679, 1992.

143. De Crespigny AJ, Wendland MF, Derugin N, Kozniewska E, Moseley ME. Real-time observation of transient focal ischemia and hyperemia in cat brain. Magn Reson Med 27:391-397, 1992.

144. Bandettini PA, Wong EC, Hinks RS, Tikofsky RS, Hyde JS. Time course epi of human brain function during task activation. Magn Reson Med 25:390-397, 1992.

145. Connelly A, Jackson GD, Frackowiak RS, Belliveau JW, VarghaKhadem F, Gadian DG. Functional mapping of activated human primary cortex with a clinical MR imaging system. Radiology 188:125-130, 1993.

146. Miller KL, Hargreaves BA, Lee J, Ress D, deCharms RC, Pauly JM. Functional brain imaging using a blood oxygenation sensitive steady state. Magn Reson Med 50:675-683, 2003.

147. Song AW, Harshbarger T, Li T, Kim KH, Ugurbil K, Mori S, et al. Functional activation using apparent diffusion coefficient-dependent contrast allows better spatial localization to the neuronal activity: evidence using diffusion tensor imaging and fiber tracking. Neuroimage 20:955-961, 2003.

148. Breger RK, Rimm AA, Fischer ME, Papke RA, Haughton VH. $\mathrm{T} 1$ and $\mathrm{T} 2$ measurements on a $1.5 \mathrm{~T}$ commercial imager. Radiology 171:273-276, 1989.

149. Fischer HW, Haverbeke YV, Schmitz-Feuerhake I, Muller RM.
The uncommon longitudinal relaxation dispersion of human brain matter. Magn Reson Med 9:441-446, 1989.

150. Wansapura JP, Holland SK, Dunn RS, Ball WS. NMR relaxation times in the human brain at 3.0T. Magn Reson Med 9:531-538, 1999.

151. Jezzard P, Duewell S, Balaban RS. MR relaxation times in human brain: measurement at 4T. Radiology 3:637-648, 1996.

152. Meyer CH, Pauly JM, Macovski A, Nishimura DG. Simultaneous spatial and spectral selective excitation. Magn Reson Med 15: 287-304, 1990.

153. Kruger G, Glover GH. Physiological noise in oxygenation-sensitive magnetic resonance imaging. Magn Reson Med 46:631-637, 2001.

154. International Electrotechnical Commission (IEC)-Medical Electrical Equipment. Particular requirements for the safety of magnetic resonance equipment for medical diagnosis, parts 2-33. Berlin, Germany, 2002.

155. Hennig J, Scheffler K. Hyperechoes. Magn Reson Med 46:6-12, 2001.

156. Mugler JP, Meyer H, Kiefer B, Wald LL, Brookeman JR. Prescribed signal evolutions: efficient 3D turbo spin-echo MR imaging with low power deposition. Electromedia, in press.

157. Wang D, Heberlein KA, LaConte S, Hu X. Inherent insensitivity to rf inhomogeneity in flash imaging. Magn Reson Med 52:927931, 2004.

158. Schmitt M, Feiweier T, Horger W, Krueger G, Schoen L, Lazar $\mathrm{RM}$, et al. Improved uniformity of RF-distribution in clinical whole body imaging at $3 \mathrm{~T}$ by means of dielectric pads. Paper presented at the 12th Annual Meeting of the International Society for Magnetic Resonance in Medicine. Kyoto, Japan, p 197, 2004

159. Katscher U, Boernert P, Leussler C, van den Brink JS. Theory and experimental verification of transmit sense. Paper presentd at the 10th Annual Meeting of the International Society for Magnetic Resonance in Medicine. Honolulu, HI, p 189, 2002. 